\title{
On the Preservation of Cooperation in Two-Strategy Games with Nonlocal Interactions
}

\author{
Ozgur Aydogmus ${ }^{1}$, Wen Zhou ${ }^{2}$ and Yun Kang ${ }^{3}$
}

\begin{abstract}
Nonlocal interactions such as spatial interaction are ubiquitous in nature and may alter the equilibrium in evolutionary dynamics. Models including nonlocal spatial interactions can provide a further understanding on the preservation and emergence of cooperation in evolutionary dynamics. In this paper, we consider a variety of two-strategy evolutionary spatial games with nonlocal interactions based on an integro-differential replicator equation. By defining the invasion speed and minimal traveling wave speed for the derived model, we study the effects of the payoffs, the selection pressure and the spatial parameter on the preservation of cooperation. One of our most interesting findings is that, for the Prisoners Dilemma games in which the defection is the only evolutionary stable strategy for unstructured populations, analyses on its asymptotic speed of propagation suggest that, in contrast with spatially homogeneous games, the cooperators can invade the habitat under proper conditions. Other two-strategy evolutionary spatial games are also explored. Both our theoretical and numerical studies show that the nonlocal spatial interaction favors diversity in strategies in a population and is able to preserve cooperation in a competing environment. A real data application in a virus mutation study echoes our theoretical observations. In addition, we compare the results of our model to the partial differential equation approach to demonstrate the importance of including non-local interaction component in evolutionary game models.
\end{abstract}

Keywords: Evolutionary game; Integro-differential replicator equations; Nonlocal interaction; Selections in genetic games.

\section{Introduction}

In nature, a population can consist of both individuals playing cooperative strategies, i.e., the cooperators (C), and individuals playing defective strategies, i.e., the defectors (D). Cooperation plays an important role in the sustainability and evolution of a population, which has been observed from cellular to population level $[7,23]$. The preservation of cooperation in a population has been extensively studied in biology, economics, and social sciences for decades $[4,10,11,20,22,23,38,42]$. It is understandable that the cooperators are prone to take a cost to assist others, while the defectors are more likely to choose selfish rational strategies to maximize one's own payoff. Cooperation is therefore expected to be rare in a population [12]. However, cooperation is critical for many complex ecosystems to sustain and evolve such as the transition from unicellular to multi-cellular organisms [38]. One of our main goals is to study the effects of nonlocal interactions

\footnotetext{
${ }^{1}$ Department of Economics, Social Sciences University of Ankara, Ankara, Turkey (ozgur.aydogmus@asbu.edu.tr)

${ }^{2}$ Department of Statistics, Colorado State University, Fort Collins, CO 80523, USA. (riczw@stat.colostate.edu)

${ }^{3}$ Sciences and Mathematics Faculty, College of Letters and Sciences, Arizona State University, Mesa, AZ 85212, USA (yun.kang@asu.edu)
} 
on the preservation of cooperation in an evolutionary setting.

Evolutionary game theory (EGT) has been applied successfully to study the evolution of populations of competing species, which provides not only a characterization of the stability of game dynamics $[4,25,44,55]$ but also a systematic approach to model and study the emergence and preservation of cooperation in a biological or social community. Traditional EGT considers $n$ competing species or strategies distributed in a population according to frequency $\boldsymbol{\rho}=\left(\rho_{1}, \cdots, \rho_{n}\right)^{\prime}$ that satisfies $\sum_{i=1}^{n} \rho_{i}=1$, and models the interactions of species or strategies by payoff matrix $\mathbf{M}=\left(m_{i j}\right)_{1 \leq i, j \leq n}$. Species or strategies with the average fitness $(\mathbf{M} \boldsymbol{\rho})_{i}$ outperforming the population average fitness $\boldsymbol{\rho}^{\prime} \mathbf{M} \boldsymbol{\rho}$ will eventually survive $[25,44,55]$. Payoff matrix $\mathbf{M}$ therefore is able to characterize the preservation of cooperation, and defines different games including the Prisoner's Dilemma (PD) game, the Snowdrift (SD) game, and the coordination game $[4,55]$. The replicator equation provides a different view of EGT. It connects the Nash equilibrium of an evolutionary game to the stable equilibrium of a corresponding dynamical system [23, 25, 55]. From the point of view of modeling, both the traditional EGT and the replicator equation focus on an infinitely large population. A framework to study the evolutionary game dynamics in finite populations was developed recently using stochastic processes. Birth and death processes in finite populations are employed by [51], and later extended to the structured populations by [47]. Deterministic population models such as the replicator equation can also be identified as the deterministic limit of these stochastic models [5]. More details on the model derivation and analysis, particularly their biological motivations, are referred to [42].

In the replicator equation setting, it is well known that the Prisoners Dilemma (PD) game is characterized by its payoff matrix $\mathbf{M}$ with defection being the only evolutionary stable strategy and the frequency of cooperators converging to zero asymptotically for any initial data in $[0,1)$. An interesting and biologically intuitive question is: can cooperation sustain in a PD game by including extra biologically reasonable factors? The first effort to answer this question is the replicator diffusion equations, which considers mobile individuals with same or different diffusion rates [21, 22, 27]. As discussed in [2, 17], the existence of traveling waves in spatially distributed moving individuals implies an invasion of a population of the defectors by cooperators. The second approach is to use the Markovian cellular automata for populations on grids. Instead of moving in space, individuals collect payoffs from a small spatial neighborhood. Simulation studies suggest that the preservation of cooperation in a PD game might be possible, see for example [45] and references therein. Another approach is to use the non-Markovian dynamics, which considers a population of individuals with memories. In this approach, history of strategies played by each player is used to calculate the transition probability among strategies [49]. These approaches provide us a perspective on potential factors that can preserve or promote cooperation strategies. However, the nonlocal interaction of population, which is important and prevailed in nature, is missing from these approaches.

In this paper, we focus on the evolutionary spatial game (ESG) by considering a population on a lattice as described by [58] and model the nonlocal spatial interactions via discretized kernels. Modifying the rate function by [53], we derive the nonlocal integro-differential replicator equation directly from microscopic rules with the aid of mesoscopic limit theory [29]. The resulting integro-differential equation is the deterministic limit of a stochastic process modeling ESG dynamics on grids in general. The model parameters include the range of nonlocal interaction, payoff matrix $\mathbf{M}$, and the natural selection pressure, on which we study the conditions to maintain the cooperation in a variety of games. We first focus on the asymptotic behavior of the derived model for PD games, and study the asymptotic tendency of the spread and invasion of the cooperators and defectors. The existence of monotone waves implies the preservation of cooperators, and therefore the preservation of cooperation in a traditional PD environment. We then extend our results to other games such as the snowdrift game (SD) and explore the effects of model parameters, along with different kernels, on the preservation of cooperation by simulation studies. Our theoretical approach naturally connects with 
the theory of traveling waves and asymptotic speed of spread (hereafter spreading speed), which was first introduced to reaction-diffusion equations by [3]. In literature, similar approaches have been employed to study game dynamics with mobile players $[22,27]$ and nonlocal epidemic or ecological models that describes the long term behavior of the population in spatial habitats $[2,31,57]$.

\section{Background and model formulations}

\subsection{Biological background}

Biologically, the models in EGT focus on interactions of individuals with observable differences. These approaches model interactions among phenotypes via the frequency dependent fitness functions, and have been used to study conditions for emergence, preservation and evolution of cooperation [42, 55]. EGT has a strong connection with the natural selection mechanism, which has been discussed in biology long before the introduction of the evolutionary game theory. Early works on this topic go back to Darvin's theory on evolution and Mendel's theory on inheritance, and the early models focus on the genetic inheritance and avoid the complex interactions.

To formulate a general frequency-dependent selection among $n$ strategies evolving in time, let $\boldsymbol{\rho}(t)=$ $\left(\rho_{1}(t), \cdots, \rho_{n}(t)\right)^{\prime}$ be the frequency of $n$ strategists, which could be different species, polynucleotide molecules, or linguistic features depending on the biological context. Denote by $\mathbf{G}(\boldsymbol{\rho})=\left(G_{1}(\boldsymbol{\rho}), \cdots, G_{n}(\boldsymbol{\rho})\right)^{\prime}$ the fitness function, then the dynamical selection model for an evolutionary process can be written as

$$
\frac{d \rho_{i}}{d t}=\rho_{i}\left(G_{i}(\boldsymbol{\rho})-\boldsymbol{\rho}^{\prime} \mathbf{G}(\boldsymbol{\rho})\right)
$$

which is a general form of the replicator equation introduced by Taylor and Jonker [52] in 1970's. Linearization of the fitness function $G_{i}(\boldsymbol{\rho})$ in terms of $\rho_{i}$ leads to the well-known simplified replicator equation. The replicator equation model plays an important role in EGT, and along its variants, has been applied in many fields including animal behaviour [36], microbial communities [8, 13, 30, 54], and plant growth [14]. Whenever $\mathbf{G}(\boldsymbol{\rho})$ on the right hand side of the equation (2.1) corresponds to the payoff matrix of the game under consideration, the evolutionary stable strategies (ESS) of the game corresponds to the locally asymptotically stable equilibria of (2.1). From the point of view of evolutionary dynamics, an ESS can be defined as a strategy that, when common in the population, cannot be invaded by any small group of individuals playing a different strategy $[36,37]$.

Also, when $G_{i}(\boldsymbol{\rho})=\sum_{j=1}^{n} m_{i j} \rho_{j}$ and $m_{i j}=m_{j i}$, that is the corresponding game is doubly symmetric, the replicator equation (2.1) is equivalent to a dynamical model of natural selection at a gene locus with $n$ alleles for diploid species [23]. Specifically, if $\rho_{i}(t)$ models the relative frequency of the allele of type $i$ in the population at time $t$ and $m_{i j}$ models the fitness of a new genotype or gene pair made from alleles of types $i$ and $j$, then the model (2.1) from EGT is also a continuous-time selection model in population genetics [19]. For this setting, the population mean fitness $\boldsymbol{\rho}^{\prime} \mathbf{G}(\boldsymbol{\rho})$ is increasing, which reflects the fundamental theorem of natural selection. Another interesting perspective is that the replicator equation (2.1) with

$G_{i}(\boldsymbol{\rho})=\sum_{j=1}^{n} m_{i j} \rho_{j}$ is equivalent to the Lotka-Volterra equation with $n-1$ species in ecology. More discussions on this topic are referred to Chapter 4 in [42].

Model (2.1), though widely employed to study unstructured populations, does not account for the nonlocal interaction and spatial heterogeneity, which are important for modeling evolutionary games. For example, the spatial interaction is a key factor to model the plants growth and expansion in ecology [14]. Another interesting example is due to [8]. They worked with two populations of E. coli whose competition is modeled 
by a PD game. One population contains a genetic element, plasmid, that helps bacteria to produce an antibacterial toxin called colicin. The other type is sensitive to colicin but has longer longevity. [8] showed that if the initial density of colicinogenic bacteria is higher than 0.02 in a well-mixed population, they can take over the whole population. Otherwise the sensitive bacteria takes over the whole population. With experiments accounting for spatial interactions, [8] reported that colicinogenic bacteria can take over the population with a lower initial frequency (only in excess of $10^{-6}$ ) and suggested that the spatial heterogeneity affects the dynamics. This study was extended to a population of E. coli with three strains by [13] and [30]. They showed that three genotypes of $E$. Coli can coexist under a spatially heterogeneous environment while a well-mixed population model predicts extinction of other two strains inaccurately.

Motivated by these, we derive the integro-differential replicator equation model from a stochastic process and microscopic rules with the aid of mesoscopic limit theory [29].

\subsection{Model derivations}

\subsubsection{Stochastic model}

Consider a population consisting of $n$ different subspecies that each of them play their own strategy. Therefore, such a population can be modeled as $n$ different strategies playing a symmetric game, whose payoff matrix is denoted by $\mathbf{M}=\left(m_{i j}\right)_{1 \leq i, j, \leq n}$. Each individual in the population is placed on a grid point $\dot{x} \in \Lambda \subset \mathbb{Z}^{d}$. We model the mutual interaction between individuals locating at $\dot{x} \neq \dot{y} \in \Lambda$ by a discrete kernel $\mathcal{J}\left(\dot{x}-y^{\prime}\right)$ satisfying $\sum_{\dot{x} \in \Lambda} \mathcal{J}(\dot{x})=1$. Each individual interacts with the remaining population according to weights assigned by kernel $\mathcal{J}$ and payoff matrix M. Let $\theta: \Lambda \rightarrow S=\{1, \cdots, n\}$ be a function whose image $\theta(\hat{x})$ is the strategy of the individual located at grid point $\dot{x} \in \Lambda$. The fitness of the individual with strategy $i$ at site $x$ is given by

$$
p(\dot{x}, \theta, i)=\sum_{\dot{y} \in \Lambda} \mathcal{J}(\dot{x}-\dot{y}) m_{i \theta(\dot{y})} .
$$

A spatial birth and death process is employed to model the dynamics. That is, an individual on $\Lambda$ is selected randomly with the sampling distribution $\operatorname{Exp}(1)$ and the current strategy $i$ is updated with respect to a non-negative rate function $r(\dot{x}, \theta, i)$ in fitness. This model was originally proposed by [58], and a similar one is constructed based on Markov generators [29].

Denote $\mathbb{I}(A)$ the indicator function of set $A$. The generator of this Markov process is then given by

$$
(L g)(\theta)=\sum_{\dot{x} \in \Lambda} \sum_{k \in S} r(\dot{x}, \theta, k)\left\{g\left(\theta^{\dot{x}, k}\right)-g(\theta)\right\},
$$

for smooth function $g$, where $\theta^{\dot{x}, k}(\dot{y})=k \mathbb{I}(\dot{y}=\dot{x})+\theta(\dot{y}) \mathbb{I}(\dot{y} \neq \dot{x})$ represents the configuration in which the agent at site $\dot{x}$ switches the current strategy to a new strategy $k$. Suppose $A \subset \mathbb{R}$ is the mesoscopic domain and $A^{\gamma}=\gamma^{-1} A \cap Z^{d}$ is the microscopic domain. Particularly, we take the limit $\Lambda \rightarrow \mathbb{Z}^{d}$ and $\gamma \rightarrow 0$ in such away that $\gamma^{-d} \approx|\Lambda| \approx n^{d}$, where $n^{d}$ denotes the size of the population and $|\cdot|$ denotes the cardinality. In addition, factor $\gamma$ is chosen in such a way that $\sum_{x} \mathcal{J}^{\gamma}(x-y) \approx \int J(x) d x=1$. We consider stochastic processes with fixed boundary conditions, and assume that the initial distribution is sufficiently regular. For example, one may assume that the distribution of initial distribution is given by a product measure with a slowly varying parameter. We refer to [29] for more details.

Cellular automaton simulations with small neighborhood assumptions have been used widely to study this model for a localized uniform kernel $\mathcal{J}$, see for example [43] and [44]. Simulation study on this model with Gaussian kernels was reported by [46]. 
Different $r(\dot{x}, \theta, i)$ have been studied in literature, for example see [29]. To mimic the replicator rule, we consider

$$
r(\dot{x}, \theta, i)=\psi(\dot{x}, \theta, i) \cdot F(p(\dot{x}, \theta, i)-p(\dot{x}, \theta, \theta(\dot{x}))),
$$

where $\psi(\dot{x}, \theta, i)=\sum_{\dot{y} \in \Lambda} \mathcal{J}(\dot{x}-\dot{y}) \mathbb{I}(\theta(\dot{y})=i)$ is the probability of finding individuals with strategy $i$ in $\Lambda \backslash\{\dot{x}\}$ with respect to kernel $\mathcal{J}$. Taking information about the payoff differences, $F(s)$ models the rate with which an individual at $\dot{x}$ adopts strategies played by others on the lattice. It is usually pre-defined; for example, one may set

$$
F(s)=\max \{0, s\}:=[s]_{+}
$$

to recover the replicator equations $[5,23]$.

\subsubsection{Mesoscopic limit}

Using results by [29], we obtain the limiting equation of the stochastic model specified by its generator (2.2) together with the rate function (2.3). Consider a continuous kernel $J(x)$ that is a probability density function on $\mathbb{R}^{d}$. It follows the mesoscopic scaling that $\mathcal{J}^{\gamma}(\dot{x}-\dot{y})=\gamma^{d} J(\gamma(\dot{x}-\dot{y}))$, where $\gamma^{-d}=|\Lambda|$ is the size of the population on $\Lambda$ [29]. For a mesoscopic domain $A \subset \mathbb{R}^{d}, A^{\gamma}=\left(\gamma^{-1} A\right) \cap Z^{d}$ is the microscopic domain. Denote $u_{i}(x, t)$ the the frequency of the $i^{\text {th }}$ strategy at location $x \in \mathbb{R}^{d}$ and time $t \in \mathbb{R}^{+}$, i.e. $\sum_{i=1}^{n} u_{i}(x, t)=1$. As $\gamma \rightarrow 0$, the microscopic domain approaches the mesoscopic continuum $A$ and an individual at $x$ interacts with increasing number of neighbors. It has been shown by [29] that the mesoscopic limit of the Markov process defined by $(\mathcal{J}, p(\dot{x}, \theta, i), r(\dot{x}, \theta, i))$ satisfies the following integro-differential equation (IDE):

$$
\frac{\partial}{\partial t} u_{i}(x, t)=\underbrace{\sum_{k=1}^{n} r(x, k, i, \mathbf{u}) u_{k}}_{\text {Relative fitness of } i^{\text {th }} \text { strategy }}-\underbrace{u_{i} \sum_{k=1}^{n} r(x, i, k, \mathbf{u})}_{\text {Relative average fitness of the population }}
$$

where $\mathbf{u}$ is an $n \times 1$ vector and $r(x, k, i, \mathbf{u}) \in \mathbb{R}$ describes how the strategy transfers from $k$ to $i$. For example, given $F(s)$ in $(2.4)$

$$
r(x, k, i, \mathbf{u})=\left\{J * u_{i}(x, t)\right\}\left[J * \sum_{l=1}^{n}\left(m_{k l}-m_{i l}\right) u_{l}\right]_{+},
$$

where $J * u_{i}=\int_{\mathbb{R}^{d}} J(x-y) u_{i}(y) d y$. Ordinary differential equation (ODE) models have been extensively used to study well-mixed populations whereas the above IDE describes the evolution of populations with nonlocal interactions among organisms in a continuum $A$.

\subsubsection{Nonlocal integro-differential replicator equation}

The traditional rate function with $F(s)$ specified in (2.4), when employed to derive an IDE, will introduce non-smooth terms to the resulting equation and also technical difficulties for analysis. On the other hand, $F(s)$ in (2.4) is a hard thresholding based on the local payoff information, which is strict in practice. We therefore consider a smoothed rate function that was introduced in [53]

$$
F(s)=1+(w s) / m_{\max },
$$

where $w \in[0,1)$ measures the strength of natural selection and $m_{\max }=\max _{i, k, p}\left|m_{k p}-m_{i p}\right|$ is the maximum fitness difference. Not only (2.6) is smooth and introduces extra flexibility, but also it is invariant under 
linear scaling of the fitness or payoff matrix. Using (2.6), the rate function (2.3) is

$$
r(x, i, k, \mathbf{u})=\left(J * u_{k}\right)\left\{1+\left(\frac{w}{m_{\max }}\right) J * \sum_{l=1}^{n}\left(m_{k l}-m_{i l}\right) u_{l}\right\} .
$$

We also notice that the mean field dynamics with (2.6) is just the replicator equation, which will be shown in Corrolary A.2. To guarantee the non-negativity of (2.7), we assume

$$
\max _{i \neq k \in S}\left\|\boldsymbol{e}_{i k} \mathbf{M}\right\|_{\infty} \leq m_{\max }
$$

where $\boldsymbol{e}_{i k}$ is a $1 \times n$ vector whose only nonzero entries are the $i^{\text {th }}$ and $k^{\text {th }}$ elements that equals to 1 and -1 , respectively.

For a given payoff matrix $\mathbf{M}$, we rescale its entries by $m_{\max }$ and still denote $m_{i j}$ the rescaled fitness hereafter. In (2.7), $J * u_{k}$ denotes the relative proportion of individuals playing strategy $k$ against remaining individuals at location $x$. Remaining terms in (2.7) are the sum of relative fitness differences between strategies $k$ and $i$. Therefore, (2.7) can be interpreted as the rate at which individuals playing strategy $i$ changes their strategies to $k$. Substituting (2.7) into (2.5) gives the nonlocal integro-differential replicator equation that for each $i$,

$$
\frac{\partial}{\partial t} u_{i}=J * u_{i}-u_{i}+w\left[\left(J * u_{i}+u_{i}\right)\left(J * \sum_{k=1}^{n} m_{i k} u_{k}\right)-\sum_{k, l=1}^{n} m_{k l}\left\{u_{k}\left(J * u_{i}\right)+u_{i}\left(J * u_{k}\right)\right\}\left(J * u_{l}\right)\right] .
$$

At $x \in \mathbb{R}^{d}$, frequencies of individuals playing different strategies evolves according to (2.9) in time. Convolutions in (2.9) essentially model the nonlocal interactions.

Several important remarks regarding model (2.9) and its interpretations are summarized below.

\section{Remark.}

1. Denote $J * \mathbf{u}$ the vector of relative frequencies of each species,

$$
\left(J * u_{i}+u_{i}\right)\left(J * \sum_{k=1}^{n} m_{i k} u_{k}\right)=\left(J * u_{i}\right)\{\mathbf{M}(J * \mathbf{u})\}_{i}+u_{i}\{\mathbf{M}(J * \mathbf{u})\}_{i},
$$

where $\{\mathbf{M}(J * \mathbf{u})\}_{i}$ models the relative payoff of species playing strategy $i$. Therefore, the fitness of species playing strategy $i$ is determined by the product of the relative payoff and the sum of the frequency and the relative frequency of individuals playing strategy $i$ at $x$. On the other hand,

$$
\sum_{k, l=1}^{n} m_{k l}\left\{u_{k}\left(J * u_{i}\right)+u_{i}\left(J * u_{k}\right)\right\}\left(J * u_{l}\right)=\left(J * u_{i}\right)\left\{\mathbf{u}^{\prime} \mathbf{M}(J * \mathbf{u})\right\}+u_{i}\left\{(J * \mathbf{u})^{\prime} \mathbf{M}(J * \mathbf{u})\right\},
$$

(2.9) can therefore be rewritten as

$$
\begin{aligned}
\frac{\partial}{\partial t} u_{i}= & J * u_{i}-u_{i}+w\left(J * u_{i}\right)\left[\{\mathbf{M}(J * \boldsymbol{u})\}_{i}-\mathbf{u}^{\prime} \mathbf{M}(J * \mathbf{u})\right] \\
& +w u_{i}\left\{(\mathbf{M}(J * \mathbf{u}))_{i}-(J * \mathbf{u})^{\prime} \mathbf{M}(J * \mathbf{u})\right\}
\end{aligned}
$$

where $\mathbf{u}^{\prime} \mathbf{M}(J * \mathbf{u})$ and $(J * \mathbf{u})^{\prime} \mathbf{M}(J * \mathbf{u})$ model the average local and nonlocal relative payoffs of the population. If the local or nonlocal average payoff of the population overwhelms the relative payoff of 
individuals playing strategy $i$, the growth rate of species playing strategy $i$ at $x$ will be negative.

2. For $w=0$, i.e. the population dynamics are independent from the fitness, (2.9) or (2.10) reduces to the nonlocal diffusion equation discussed in [1]. For $w>0$, the payoff matrix and the underlying game influence the population dynamics.

3. Consider the spatially homogeneous version of rate function (2.7)

$$
r(i, k, \boldsymbol{\rho})=\rho_{k}\left\{1+w \sum_{l=1}^{n}\left(m_{k l}-m_{i l}\right) \rho_{l}\right\},
$$

where the $k^{\text {th }}$ entry of $\boldsymbol{\rho}$ denotes the frequency of the species playing the $k^{\text {th }}$ strategy (analog to $u_{k}$ ). Then, using rate function (2.7), we can recover the well-known replicator equation

$$
\frac{d \rho_{i}}{d t}=2 w \rho_{i}\left(\sum_{k=1}^{n} m_{i l} \rho_{l}-\sum_{k, l=1}^{n} m_{k l} \rho_{k} \rho_{l}\right) .
$$

Therefore, (2.9) or (2.10) is essentially a spatial generalization of (2.11) that incorporates effects of spatially nonlocal interactions into the finesses of the underlying game; while (2.11) assumes a uniform population distribution and is a special case of the dynamical model (2.1). Another important difference between (2.9) or (2.10) and (2.11) is that the selection pressure constant $w$ does not affect the dynamics in the later one while it changes the dynamics in the former one due to nonlocal term $J * u-u$.

At last, we collect some fundamental properties for (2.9) or (2.10) and (2.11) are below.

Proposition 1. The following statements hold for (2.9) and (2.11) provided the existence of the corresponding solutions.

(I) Adding an arbitrary constant to all entries of a column of the payoff matrix $\mathbf{M}$ does not change the dynamics of replicator equations.

(II) Constant solutions to (2.9) is also a constant solution to (2.11), and vice versa.

(III) Given $u(x, 0) \in[0,1]$, we have $0 \leq u(x, t) \leq 1$ and $\sum_{i=1}^{n} u_{i}(x, t)=1$ for all $t>0$ and spatial locations $x \in R^{d}$.

Property (I) is readily obtained from the definition of above rate function (2.7) and helps to simplify the parameter space along with (2.8). As for (2.11), it implies that it is the relative difference between payoff values rather than the payoff values themselves playing a critical role in the evolutionary game dynamics. Property (II) ensures that all Nash equilibria of the classical game (without spatial nonlocal interactions) with payoff matrix $\mathbf{M}$ are also constant solutions to (2.9) or (2.10). Property (III) is an immediate consequence of Theorem A.1 by [29] and it assures that the game simplex stays invariant.

\section{The impact of spatially nonlocal interactions on the preservation of cooperation}

When some strategy invades a new habitat, its corresponding population size grows and its spatial range expands. As discussed in $[24,41]$, the rate at which the range expands is called the invasion speed and serves as a basic descriptive statistic for invasion dynamics. Furthermore, as defined in [40], whenever the linear determinacy condition holds, the critical invasion speed is equal to the minimal traveling wave speed, see, for example, [24] and references therein. 
Spatially nonlocal interactions provide more flexibility to the underlying game dynamics so that the cooperation can sustain more likely than traditional games. To explore this intuition, we study existence of traveling wave solutions and asymptotic speed of propogation for two-strategy games modeled by (2.9) in this section. Following Aronson [2], we consider an habitat containing individuals playing both strategies.

\subsection{Nonlocal integro-differential replicator equation for two-strategy games}

Hereafter, we focus on one-dimensional two-strategy games, that is $\mathbf{u}=\left(u_{1}, u_{2}\right)^{\prime}$ and $d=1$. Therefore, there are only two possible strategies, strategy 1 and 2 , in the population, whose frequencies correspond to $u_{1}$ and $u_{2}$, respectively. Specifically, for a PD game, those two strategies are defection and cooperation, respectively. Games with a dominated strategy correspond to either PD or harmony games, games with coexisting strategies correspond to the SD games that are also known as Hawk-Dove games, and remaining are known as the coordination games. The aim of this section is to give some basic definitions and assumptions to study traveling waves and spreading speeds.

By Proposition 1, one may consider the following matrix for two-strategy games without losing generalities

$$
\mathbf{M}=\left[\begin{array}{ll}
1 & 2 \\
\alpha & 0 \\
0 & \beta
\end{array}\right] \quad \begin{aligned}
& 1 \\
& 2
\end{aligned}
$$

where $|\alpha|,|\beta| \leq 1$ and $|\alpha|=1$ or $|\beta|=1$. Denote $u:=u_{1}=1-u_{2}$ the frequency of the second strategy in the population, our model (2.9) reduces to

$$
\frac{\partial}{\partial t} u=H[u]:=J * u-u+w(u+J * u-2 u J * u)\{(\alpha+\beta) J * u-\beta\} .
$$

Equation (3.1) is called monostable if the corresponding ordinary differential equation (3.17), which models the spatially homogeneous population, has only two equlibria $u=0$ and $u=1$, and only one of them is stable. Equation (3.1) is called bistable if there exists two stable equilibria. It is easy to see that (3.1) is monostable for the PD and Harmony games, while it is bistable for the coordination game. Equation (3.1) is called quasi-monotone if its right hand side is increasing in $J * u$. Linearization of $H[u]$ at $u=0$ gives

$$
\mathcal{M}[u]=-(1+w \beta) u+(1-w \beta)(J * u)
$$

and (3.1) is linearly determinate if it satisfies the growth condition that for each $\epsilon>0$, there exists a $\delta>0$ such that

$$
(1-\delta) \mathcal{M}[u] \leq H[u] \leq \mathcal{M}[u]
$$

for $0 \leq u \leq \epsilon$.

The kernel $J$ is designated for modeling the nonlocal interaction and we assume it satisfies the following assumptions.

(J1) $J \geq 0$ and $\int_{\mathbb{R}} J(y) d y=1$, i.e. the kernel is essentially a probability density function.

(J2) For the moment generating function (MGF) of $J$

$$
M(s)=\int_{\mathbb{R}} e^{s y} J(y) d y,
$$

we assume that the $k^{\text {th }}$ derivative of the moment generating function, $M^{(k)}(s)$, exists for $k=0,1,2$ whenever $-\infty<s<\infty$. 
(J3) Define $A(s):=\left(d_{1} M(s)-d_{2}\right) / s$ with $d_{1}, d_{2} \in \mathbb{R}^{+}$, we assume that $A(s)$ is increasing on $\left(-\infty, s_{0}^{-}\right) \cup$ $\left(s_{0}^{+}, \infty\right)$ and decreasing on $\left(s_{0}^{-}, 0\right) \cup\left(0, s_{0}^{+}\right)$for some $s_{0}^{ \pm}$. Existence of such $s_{0}^{ \pm}$is discussed in Section A.7.

\subsection{Evolutionary spatial PD games}

Consider individuals playing a PD game on an unbounded continuous domain, where those playing the defective strategy are called the defectors and others are called the cooperators, respectively. To describe the invasion of one strategy to a habitat occupied by another strategy, or more precisely, individuals playing that strategy, we study the speed of population expansion based on the existence of traveling wave solutions and spreading speeds to the underlying nonlocal replicator equation. To study the minimal speed for traveling wave solutions to (3.1) in a PD game, we consider payoff matrix $\mathbf{M}$ with $\alpha>0$ and $\beta<0$ and natural selection parameter $w$ satisfying the following assumptions.

(P1) The quasi-monotonicity condition is held for (3.1) whenever $w(2 \beta+\alpha)>-1$, see Section A.2 for technical details. For $\alpha=1$, it automatically holds for any $w \in[0,1)$; while $\sup _{w \in[0,1)}\{w: w \leq-1 /(-2+\alpha)\} \downarrow$ $1 / 2$ as $\alpha$ decreases for $\beta=-1$. Thus, this condition is valid for any pairs of $(\alpha, \beta)$ provided $w<1 / 2$. Hence, a weak selection with small $w$ prevents extreme PD games that the selection of defection is less favored when the defection's fitness is very large compared to that of the cooperation.

(P2) The linear determinacy condition is held for (3.1) whenever $\alpha+\beta \leq 0$, i.e. $\beta=-1$. This condition is first employed to show the existence of traveling wave solutions, and we will relax it to obtain similar results by studying asymptotic speed of propagation.

\subsubsection{Existence of Traveling Waves}

Under Condition (P2), we define the minimal traveling wave speeds in both positive and negative directions as a function of payoff values, natural selection parameter $w$ and nonlocal kernel $J$, and show their existence using results by [50]. First, consider the linearized (3.1), $\partial u / \partial t=\mathcal{M}[u]$. We introduce the traveling wave coordinate $\xi=x+c t$, where $c$ denotes the traveling wave speed. For $U(\xi)=u(x, t)$, we have

$$
c U^{\prime}(\xi)=-(1+w \beta) U(\xi)+(1-w \beta)(J * U) .
$$

Consider standard ansatz $U(z)=e^{s z}$ in $(3.2)$, the characteristic equation is

$$
\Delta_{c}^{0}(s):=-c s+(1-w \beta) M(s)-(1+w \beta)=0 .
$$

Assumptions (J1) and (J2) imply that $\Delta_{c}^{0}$ is convex and $\Delta_{c}^{0}<0$ for sufficiently small (large) $c$ and negative (positive) $s$, so that it admits two zeros. Also, $\Delta_{c}^{0}$ has double root for some $c_{0}^{ \pm}$. We can therefore define the right and left minimal traveling wave speeds by

$$
\begin{aligned}
& c_{0}^{+}=\inf _{s>0} \frac{-(1+w \beta)+(1-w \beta) M(s)}{s}, \\
& c_{0}^{-}=\sup _{s<0} \frac{-(1+w \beta)+(1-w \beta) M(s)}{s} .
\end{aligned}
$$

Equations (3.3) and (3.4) are well-defined under Assumption (J2).

To (3.1), the traveling wave solution $U(\xi)$ satisfies

$$
c U^{\prime}=J * U-U+w(U+J * U-2 U J * U)((\alpha+\beta) J * U-\beta)
$$


with boundary conditions

$$
\begin{aligned}
& U(-\infty)=0 \text { and } U(\infty)=1 \text { for } c>c_{0}^{+}, \\
& U(-\infty)=1 \text { and } U(\infty)=0 \text { for } c<c_{0}^{-} .
\end{aligned}
$$

Given (P2), all nonlinear terms in (3.5) are negative so that $c_{0}^{ \pm}$are the upper and lower bounds of the wave speeds for original nonlocal replicator equation (3.1), respectively. We will show that as long as (P2) holds, $c_{0}^{ \pm}$are also minimal traveling wave speeds for (3.1). The following theorem establishes the existence and non-existence of traveling wave solutions in terms of minimal traveling wave speeds.

Theorem 1. Suppose kernel J satisfies (J1)-(J2), and Conditions (P1)-(P2) hold. A monotone solution $U \in[0,1]$ to $(3.5)$ and $(3.6)$ exists only for $c \notin\left(c_{0}^{-}, c_{0}^{+}\right)$where $c_{0}^{-}, c_{0}^{+}$are non-zero real numbers defined in (3.3), (3.4), respectively.

Theorem 1 is proved in Appendix A.4. Biologically, the spread of cooperators takes place via the propagation of a population front separating the regions where the defectors are present from the regions where cooperators are present with a considerable density. Therefore, the solutions describing the propagation of traveling waves, which are $u(x, t)=U(\xi)$ with $c$ the speed of wave, characterizes such spread. That is, the solution switches from the equilibrium state $u=0$ to the equilibrium state $u=1$ as interpreted in [21]. Also, no such solutions exist for $c \in\left(c_{0}^{-}, c_{0}^{+}\right)$. Therefore, the existence of traveling waves reflects the preservation of cooperation and the non-existence of them implies that defectors take over the population.

Furthermore, the monotonicity implies that the only possible type of traveling wave described by (3.5) is a propagating front connecting the lower and the upper steady states, which are $u=0$ and $u=1$; see Figure 1. The minimal speed of the wave strongly depends on the fitness parameters, the selection pressure and the interaction kernel as seen from (3.3) and (3.4), which give the lowest and highest possible value of the wave speed for biological spread of the cooperation to take place.

Corollary 1. Consider a spatial evolutionary PD game with two strategies, cooperation and defection, where the spatial nonlocal interaction can be modeled by a kernel $J$ satisfying (J1)-(J2). Then, under the weak natural selection condition, (P1), cooperation can sustain whenever $c \notin\left(c_{0}^{-}, c_{0}^{+}\right)$.

\subsubsection{Without linear determinacy: spreading speeds}

Though providing technical conveniences, Condition (P2) lacks the biological intuitions. We employ the theory on spreading speeds developed by [32] to establish the main result of this section. In particular, we show that there exist an asymptotic speed of propagation and it coincides with the minimal traveling wave speed whenever linear determinacy is satisfied. As pointed out by [32], the spreading speed at which one subspecies invades the territory of an established set of other subspecies can be characterized as the lowest speed of a suitable family of traveling waves to the underlying model. We can therefore establish similar results to Theorem 1 without Condition (P2).

Let operator $\mathcal{F}$ be

$$
\mathcal{F}[u](x, t)=(1-w \beta)(J * u)+2 w \beta u(J * u)+w(\alpha+\beta)(J * u)\{u+(J * u)-2 u(J * u)\},
$$

(3.1) is reduces to

$$
\frac{\partial}{\partial t} u(x, t)=-(1+w \beta) u(x, t)+\mathcal{F}[u](x, t) .
$$

The following result implies that the defectors invade the population of cooperators for some spreading speeds without Condition (P2) provided that a part of spatial habitat is preoccupied by the defectors. Whenever 
Condition (P2) holds, the minimal traveling wave speeds agree with the spatial propagation speed for the model with nonlocal interactions.

Theorem 2. Suppose that Conditions (J1)-(J3) and (P1) hold and the initial function $u_{0}=u(x, 0)$ is 0 for all sufficiently large $x$, and there are constants $0<\sigma \leq \rho<1$ such that $0 \leq u_{0} \leq \rho$ for all $x$ and $u_{0} \geq \rho$ for all sufficiently negative $x$. Then, there exists $c_{+}^{*}$ for which the solution u to (3.7) satisfies

$$
\lim _{t \rightarrow \infty} \sup _{x \geq t\left(c_{+}^{*}+\epsilon\right)} u(x, t)=0
$$

and

$$
\lim _{t \rightarrow \infty} \sup _{x \leq t\left(c_{+}^{*}-\epsilon\right)}\{1-u(x, t)\}=0
$$

for any $\epsilon>0$. That is, the defectors spreads at a speed no higher than $c_{+}^{*}$. Moreover, $c_{0}^{+}=c_{*}^{+}$provided Condition (P2) is satisfied.

Theorem 2 follows from [32, Theorem 2.1], for which we provide a detailed discussion in Appendix A.5. Here, $c_{*}^{+}$is the spreading speed of the defectors along the positive direction, which is defined by (A.6) in Appendix A.5. These definitions imply that the defectors invade the population if the spreading speed is no larger than $c_{*}^{+}$. Otherwise, the cooperators will preserve. When the initial condition is changed accordingly, one can also define the spreading speed in the negative direction $c_{*}^{-}$as specified by (A.7). Also, given Condition (P2), $c_{0}^{-}$in (3.4) specifies the negative of the leftward spreading speed and $c_{0}^{-}=-c_{*}^{-}$, which is analogous to that for $c_{0}^{+}$and $c_{*}^{+}$.

Theorem 2 connects the minimal traveling wave speed and the spreading speed that they are same as long as Condition (P2) is satisfied, i.e. $\alpha \leq-\beta$, which only depends on $\operatorname{sign}(\alpha+\beta)$. Otherwise, the spreading speed is always greater than or equal to the minimal traveling wave speed. Particularly, Theorem 2 implies that, based on the proposed IDE model, in an evolutionary spatial PD game the defectors spread at a spreading rate $c<c_{*}^{+}\left(>c_{*}^{-}\right)$and the cooperators will take over the population for some initial conditions whenever the spreading rate $c$ is larger (smaller) than $c_{*}^{+}\left(c_{*}^{-}\right)$. Lastly, we notice that for the Harmony game, ESG model (2.9) is monostable given $\alpha<0$ and $\beta>0$. Therefore, results for the PD game can be easily applied to the Harmony game by taking $u(x, t)$ as the frequency of the cooperators at $(x, t)$.

Remark. The existence of the traveling waves, without Condition (P2), can be derived using a different techniques developed by [16]. We state the theorem as follows and provide its proof in Appendix A.6.

Theorem 3. Suppose that Conditions (J1)-(J3) and (P1) hold. Then the solution semi-flow generated by equation (3.7) admits a spreading speed $c_{+}^{*}$ which is also the minimal wave speed for monotone traveling waves connecting 1 and 0 . Further, the obtained traveling waves are also classical solutions to (3.7).

\subsection{Evolutionary spatial SD games}

For an SD game where $\alpha, \beta<0$, which is also known as the Hawk-Dove game, the coexistence of players using strategy 1 and 2 is the ESS in the traditional spatially homogeneous population settings. Hence, for a spatially homogeneous SD game, there exist two unstable equilibria 0 and 1 and one stable non-trivial equilibria, $e=\beta /(\alpha+\beta) \in(0,1)$. In contrast, evolutionary spatial SD games studied in this paper are more flexible and allow a pure population, which consist of either the first or second strategy, to be the evolutionary outcome of the dynamic processes. 


\subsubsection{Traveling wave solutions to the evolutionary spatial SD game}

For an evolutionary spatial SD game, the existence of traveling wave solutions connecting the stable and unstable equilibria can be established using similar arguments in Section 3.2. For the equilibria 0 and $1-e$, we consider the integro-differential replicator equation for the frequency $v$ of individuals playing strategy 2 as follows:

$$
\frac{\partial}{\partial t} v=(J * v)-v+w\{v+(J * v)-2 v(J * v)\}\{(\alpha+\beta)(J * v)-\alpha\} .
$$

Note that (3.1) and (3.10) are linearly determined for the parameter region of SD game. Similarly to (3.3) and (3.4), we define traveling wave speeds for individuals playing strategy 1 by

$$
\begin{aligned}
& c_{1}^{+}=\inf _{s>0} \frac{-(1+w \alpha)+(1-w \alpha) M(s)}{s}, \\
& c_{1}^{-}=\sup _{s<0} \frac{-(1+w \alpha)+(1-w \alpha) M(s)}{s} .
\end{aligned}
$$

Here we would like to note that both equations (3.1) and (3.10) are linearly determinate for SD games. Define the following hyper-polygon in $\mathbb{R}^{3}$ :

$$
\Gamma=\{\alpha, \beta \in(-1,0), w \in[0,1): w(2 \alpha+\beta)>-1, w(2 \beta+\alpha)>-1\}
$$

representing the parameter region for which equations (3.1) and (3.10) are quasi-monotone. We have the following theorem for the existence of traveling waves for an evolutionary spatial SD game.

Theorem 4. Suppose that $J$ satisfies (J1)-(J2) and assume $(\alpha, \beta, w) \in \Gamma$.

1. A monotone solution $U \in[0, e]$ to (3.5) exists only for $c \notin\left(c_{0}^{-}, c_{0}^{+}\right)$.

2. A monotone solution $V \in[0,1-e]$ to the traveling wave equation corresponding to (3.10) exists only for $c \notin\left(c_{1}^{-}, c_{1}^{+}\right)$.

The proof of Theorem 4 is similar to the proof of Theorem 1 that is detailed in Appendix A.4.

\subsubsection{Asymptotic behaviors}

Analogous to Theorem 2, we obtain the asymptotic behavior of the frequencies of individuals playing strategies 1 and 2 in an evolutionary spatial SD game following the classical theory [2,35]. On the moving coordinate $\xi=x+c t,(3.7)$ reduces to

$$
\frac{\partial}{\partial t} u(\xi, t)=Q_{c}[u(\xi, t)]:=-c u_{\xi}-(1+w \beta) u+\mathcal{F}[u] .
$$

The existence, uniqueness and monotonicity of solutions to (3.7), which are shown in Section A.3, are also valid for (3.13). The next critical proposition states that the strategy 1 with a small initial frequency can invade the population of strategy 2 for $c \in\left(c_{0}^{-}, c_{0}^{+}\right)$. Therefore, the evolutionary outcome is the strategy 1 for $c \in\left(c_{0}^{-}, c_{0}^{+}\right)$. On the other hand the strategy 1 cannot spread if $c \notin\left[c_{0}^{-}, c_{0}^{+}\right]$

Proposition 2. Assume (J1)-(J3) and assumptions of Theorem 4 are satisfied. For any $\xi \in \mathbb{R}$, we have

(i). for any initial condition $u(x, 0)$ that is nonzero in a bounded interval, solution $u$ to the initial value problem (3.13) satisfies

$$
\lim _{t \rightarrow \infty} u(\xi, t)=e \text { or } 1 \text { if } c \in\left(c_{0}^{-}, c_{0}^{+}\right),
$$


with $c_{0}^{ \pm}$defined by (3.3) and (3.4); and

(ii). for any initial condition $v(x, 0)$ that is nonzero in a bounded interval, solution $v$ to the initial value problem (3.10) satisfies

$$
\lim _{t \rightarrow \infty} v(\xi, t)=1-e \text { or } 1 \text { if } c \in\left(c_{1}^{-}, c_{1}^{+}\right),
$$

with $c_{1}^{ \pm}$defined by (3.11) and (3.12).

The proof of $(i)$ in Proposition 2 follows from Lemma A.1, whose proof is detailed in Appendix A.7, and (ii) can be shown similarly. Suppose that $c \in\left(c_{0}^{-}, c_{0}^{+}\right) \cap\left(c_{1}^{-}, c_{1}^{+}\right)$. By $(i)$ in Proposition 2 and the comparison principle, we have $u \rightarrow e$ or 1 when $u(x, 0) \neq 0$ in some interval $\mathcal{I}$. Similarly, $v=1-u \rightarrow 1-e$ or 1 as $t \rightarrow \infty$. These imply that $u \rightarrow e$ as $t \rightarrow \infty$, which is summarized in the following corollary.

Corollary 2. Suppose that $c \in\left(c_{0}^{-}, c_{0}^{+}\right) \cap\left(c_{1}^{-}, c_{1}^{+}\right)$and both initial conditions $u_{0}$ and $v_{0}$ are nonzero in some sets containing intervals. Then, solution $u$ to the initial value problem (3.13) satisfies

$$
\lim _{t \rightarrow \infty} u(\xi, t)=e
$$

\subsubsection{A phase transition for the evolutionary spatial SD game}

As functions of game parameters $\alpha$ and $\beta$, it is easy to see that $c_{0}^{+}<c_{1}^{+}$and $c_{0}^{-}>c_{1}^{-}$given $\alpha<\beta$. For $\beta>\alpha$, it admits either $\left(c_{0}^{-}, c_{0}^{+}\right) \subset\left(c_{1}^{-}, c_{1}^{+}\right)$or $\left(c_{1}^{-}, c_{1}^{+}\right) \subset\left(c_{0}^{-}, c_{0}^{+}\right)$. As a result, $c_{0}^{+}$and $c_{1}^{-}$provide a partition of the parameter space. Interestingly, a phase transition about the asymptotic behavior of $u$ is observed with respect to $c_{0}^{+}$and $c_{1}^{-}$.

In fact, by results in [39], the comparison principle, and the linear determinacy of (3.1) and (3.10), we have

$$
u(x, t) \leq \lambda e^{-s\left(x-c_{0}^{+} t\right)}
$$

provided $u(x, 0) \leq \lambda e^{-s x}$ for some $\lambda>0$. This implies that $u(x+c t, t) \rightarrow 0$ as $t \rightarrow \infty$ for $c>c_{0}^{+}$. Applying the same argument to $v(x, t)$, one can show that $v(x+c t, t)=1-u(x+c t, t) \rightarrow 0$ as $t \rightarrow \infty$ for $c<c_{1}^{-}$. Therefore, the following result summarizes this phase transition for evolutionary spatial SD game when the half plane is preoccupied by the strategy 1 and the other half is occupied by the strategy 2 .

Theorem 5. Assume $J$ satisfies (J1)-(J3), and assumptions in Theorem 4 hold. If the negative half space is preoccupied by the strategy 1 while the remaining domain is occupied by the strategy 2 , then

$$
\lim _{t \rightarrow \infty} u(\xi, t)= \begin{cases}0 & \text { if } c>c_{0}^{+} \geq c_{1}^{-}, \\ e & \text { if } c_{0}^{+}>c>c_{1}^{-}, \\ 1 & \text { if } c_{0}^{+} \geq c_{1}^{-}>c,\end{cases}
$$

where $u$ is the solution to (3.1).

Results in Theorem 5 suggest that there are three possible outcomes of an evolutionary spatial SD game: either individuals playing the strategy 1 or 2 will take over the whole population, or the two groups coexist, which depends on the spreading speeds.

Remark. Results in (3.16) hold for any initial conditions satisfying exponential bounds. For compactly supported initial condition, some extra simulation results are presented in Appendix B. 


\subsection{Evolutionary spatial coordination games}

A coordination game, in contrast to the SD games, favors pure strategies that the corresponding replicator system becomes bistable and admits an unstable interior equilibrium that reflects the unstable coexistence of both strategies 1 and 2. In contrast, an evolutionary spatial coordination game with nonlocal interactions may allow the coexistence to be an evolutionary outcome. Using results by [9] and Theorem 5 in [29], we have the following theorem for the existence of traveling waves to the evolutionary spatial coordination game.

Theorem 6. Suppose $J$ satisfies (J1)-(J2). There exists a function $U \in C^{1}(\mathbb{R})$ satisfying $U^{\prime}(\xi)>0$ and $\lim _{|\xi| \rightarrow \infty} U^{\prime}(\xi)=0$ with $\xi=x+$ ct is the solution to (3.5) with boundary conditions

$$
U(-\infty)=0 \text { and } U(+\infty)=1,
$$

and $U$ connects equilibria 0 and 1 . In addition, it is unique up to translations.

Hence, along the traveling wave solution of the IDE for coordination games, the dominating equlibrium drives out others. The dominating one can be either of the two strategies, 1 or 2 , depending on the minimal traveling wave speed $c$. For example, if the kernel is a symmetric function of its argument the dominating equlibrium is $u=0$ if $c<0$ and $u=1$ if $c>0$.

\subsection{Summary}

For a payoff matrix $\mathbf{M}$ as given in Section 3.1, the replicator equation for traditional games with spatially homogeneous populations is

$$
\frac{d \rho}{d t}=f(\rho):=\rho(1-\rho)\{(\alpha+\beta) \rho-\beta\} .
$$

In Table 1, we summarize the stability for different ESGs and compare their evolutionary outcomes with the evolutionary outcomes of traditional games modeled by (3.17). Intuitively, the nonlocal spatial interaction in ESGs has promoted diversities of the equilibrium in game dynamics. They allow the equilibria, which were originally unstable in the traditional game with well-mixing population, to survive with the aid of spatial freedoms. Particularly, in the PD games, the cooperation will be preserved given nonlocal spatial interactions, as we speculated before.

Table 1: Comparison of evolutionary outcomes between the traditional games and ESGs.

\begin{tabular}{lll}
\hline Game & Homogeneous game (3.17) & ESG (3.1) \\
\hline SD Game & $\begin{array}{l}\text { The coexistence of two strate- } \\
\text { gies, } 1 \text { and 2, is the only stable } \\
\text { equilibrium. }\end{array}$ & $\begin{array}{l}\text { All three scenarios may be evolu- } \\
\text { tionary outcomes of the dynamic } \\
\text { model depending on } c .\end{array}$ \\
PD Game & $\begin{array}{l}\text { Only the defection (strategy 1) is } \\
\text { evolutionary (and also globally) } \\
\text { stable. }\end{array}$ & $\begin{array}{l}\text { If } c \notin\left(c_{*}^{-}, c_{*}^{+}\right) \text {, cooperation (strat- } \\
\text { egy } 2) \text { drives out defection; other- } \\
\text { wise, the defection (strategy 1) takes } \\
\text { over the whole habitat. }\end{array}$ \\
Coordination Game & $\begin{array}{l}\text { Either the strategy 1 or strategy } \\
2 \text { is stable. }\end{array}$ & $\begin{array}{l}\text { Either strategy } 1 \text { or strategy 2 may } \\
\text { win depending on players' speed } c .\end{array}$ \\
\hline
\end{tabular}




\section{Simulation Studies}

In this section, we turn to simulation studies on the effects of model parameters on the preservation of cooperation in evolutionary spatial games discussed in Section 3. The parameters of interest are the game parameter $(\alpha, \beta)$, the natural selection parameter $w$, and the nonlocal interaction kernel $J$.

\subsection{Evolutionary spatial PD games}

We consider an evolutionary spatial PD game whose payoff matrix is obtained from an experiment on the selection of RNA bacteriophage $\Phi 6$ and $\Phi \mathrm{H} 2$ [54]. More details of the experiment is discussed in Section 5. For demonstration, we set $w=0.5$ and $J(x)=e^{-\sigma^{2} x^{2} / 2} / \sqrt{2 \pi \sigma^{2}}$ with $M(s)=e^{\sigma^{2} s^{2} / 2}$. For $\sigma=0.5,\left(c_{0}^{-}, c_{0}^{+}\right)=(-0.784,0.784)$; and for $\sigma=2,\left(c_{0}^{-}, c_{0}^{+}\right)=(-3.134,3.134)$. Theorem 1 implies that given $c=-1.5$, traveling wave solutions to (3.1) do not exist for $\sigma=2$, while there does exist traveling wave solutions for $\sigma=0.5$. Panel (a) in Figure 1 shows the formation of a traveling wave whose front towards the negative direction as time evolves, and panel (b) suggests that no traveling waves along the negative direction are formed and the solution converges to constant 1 on the domain. Therefore, cooperation sustains in panel (a) in Figure 1.

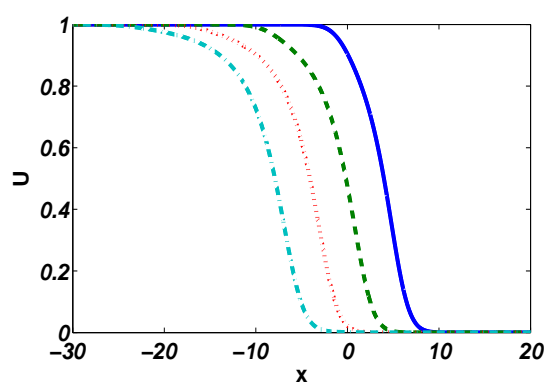

(a) Traveling wave solutions for $\sigma=0.5$

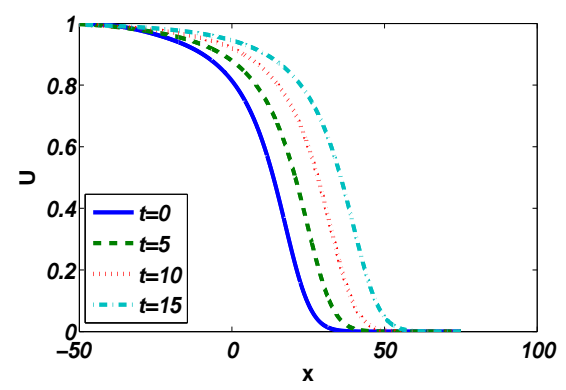

(b) Non-existence of traveling wave solutions for $\sigma=2$

Figure 1: Panels (a) and (b) display the existence and non-existence of traveling waves of an evolutionary PD game. The underlying game has payoff $\alpha=0.181, \beta=-1$ [54]. We set $w=0.369$ and $c=-1.5$. Kernel $J$ is centered Gaussian with standard deviation $\sigma$ (the range parameter). Successive waves are separated by 5 time units; for simulations, $\Delta x=0.002$ on $[-100,100]$ and $\Delta t=0.0005$.

\subsubsection{Effects of parameters on the preservation of cooperation}

Theorem 1 implies the existence of invasion waves connecting two pure equilibria whenever the wave speed satisfies $c \notin\left(c_{0}^{-}, c_{0}^{+}\right)$. Thus, $c_{0}^{+}-c_{0}^{-}$measures the likelihood that cooperation is preserved in a population that is, the cooperation is favored when $c_{0}^{+}-c_{0}^{-}$is small. For model $(3.1), c_{0}^{+}-c_{0}^{-}$is a function of the game parameter $(\alpha, \beta)$, the natural selection parameter $w$, and the kernel $J$. For the kernel $J,\left.M^{\prime \prime}(s)\right|_{s=0}$ quantifies the variability and models the strength of spatially nonlocal interactions. For following discussion, we denote $\sigma:=\left(\left.M^{\prime \prime}(s)\right|_{s=0}\right)^{1 / 2}$. Through examining (3.3) and (3.4), we observe:

1. For the payoff matrix $\mathbf{M}$, only $m_{22}=\beta$ influences $c_{0}^{+}-c_{0}^{-}$provided $(\mathrm{P} 2)$ that is, $c_{0}^{+}-c_{0}^{-}$increases as $\beta$ decreasing. Hence, large $\beta$, i.e. a less extreme $\mathrm{PD}$ game, favors cooperation.

2. Likewise, small natural selection intensity $w$ favors cooperation. 
3. For a centered Gaussian $J$ with standard deviation $\sigma,(3.3)$ and (3.4) implies small $\sigma$ favors cooperation, i.e. a "localized" evolutionary spatial PD game favors cooperators. Similar numerical results are obtained using cellular automaton (see eg. [46]).

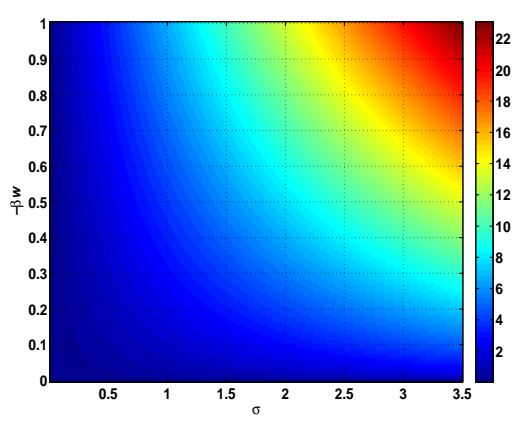

(a) Centered Gaussian

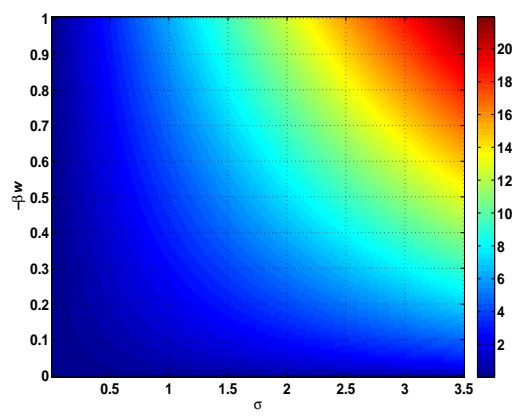

(b) Centered Uniform

Figure 2: Effects of $\sigma$ and $w \beta$ on the length of interval $\left(c_{0}^{-}, c_{0}^{+}\right)$.

To further investigate effects of $w \beta$ and $\sigma$ on $c_{0}^{+}-c_{0}^{-}$, we study a two kernel families. Heat maps in Figure 2 display $c_{0}^{+}-c_{0}^{-}$as a function of $w \beta$ and $\sigma$ for centered Gaussian (G) and centered uniform (U) $(J(x)=I(|x| \leq \sqrt{3} \sigma) /(2 \sqrt{3} \sigma))$, respectively. From Figure $2, c_{0}^{+}-c_{0}^{-}$increases in $\sigma$ and $-w \beta$, so that defectors are favored by long range spatial interactions and small $w \beta$, which is due to a strong natural selection or an extreme PD game biased towards defectors.

Furthermore, consider set $\mathcal{E}_{\epsilon}(J)=\left\{(\sigma, w \beta):\left(c_{0}^{+}-c_{0}^{-}\right)(J)<\epsilon\right\}$ for fixed $\epsilon>0$, which is the subset in parameter space that gives $c_{0}^{+}-c_{0}^{-}<\epsilon$, which is in favor of cooperation. Hence, a large $\mathcal{E}_{\epsilon}(J)$ indicates that cooperation is preferable by the evolutionary spatial game indexed by the kernel $J$. Figures 2 and 3 imply that cooperation is favored by the centered Gaussian kernel in contrast to the centered uniform kernel, i.e. $\mathcal{E}_{\epsilon}(G) \subset \mathcal{E}_{\epsilon}(U)$ for a common $\epsilon>0$. This, in fact, agrees with the concentration properties of the

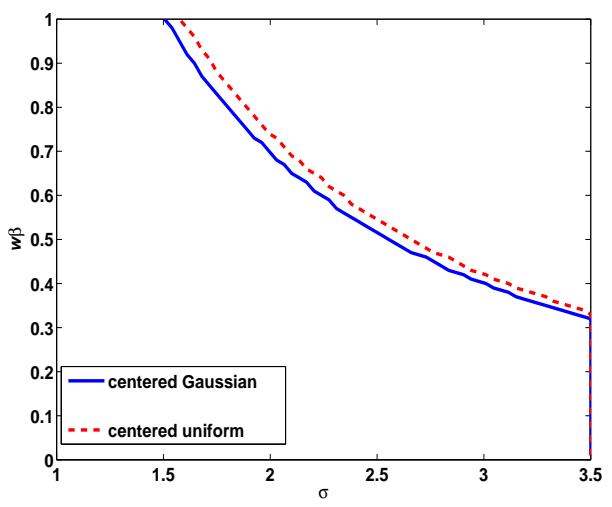

Figure 3: Boundaries of $\mathcal{E}_{\epsilon}(J), \partial \mathcal{E}_{\epsilon}(J)$, for a variety of kernels with $\epsilon=10$. The closer the $\partial \mathcal{E}_{\epsilon}(J)$ to the origin, the smaller the $\mathcal{E}_{\epsilon}(J)$ and therefore the more "hostile" the underlying evolutionary spatial game against cooperation. For symmetric kernels, $\partial \mathcal{E}_{\epsilon}(J)=\left\{(\sigma,-w \beta):(1-w \beta) M^{\prime}\left(s^{*}\right)=\epsilon / 2\right\}$ where $s^{*}$ is the solution to $M^{\prime}(s) s-M=-(1+w \beta) /(1-w \beta)$.

two kernels. With respect to the whole domain, the centered uniform kernel is more concentrated than the 
Gaussian kernel and therefore models more localized interactions. Thus it favors cooperation more than the Gaussian kernel.

\subsubsection{Spreading speeds}

As mentioned in Section 3.2.2, one can observe that critical spreading speeds $c_{*}^{ \pm}$may not be equal to critical wave speeds $c_{0}^{ \pm}$if we do not assume the linear determinacy of the corresponding monostable equation. Thus minimal speed in a spatial PD game does not just depend on parameters $w$ and $\beta$ but also it is affected by $\alpha$.

To be able to illustrate the effect of parameter $\alpha$ in a spatial PD game, we consider the following model parameters $\beta=-1$ and $w=0.1$, and the players' speed $c=-0.075$. Kernel $J$ is the centered Gaussian with standard deviation $\sigma=0.1$. The corresponding minimal wave speed is $c_{0}^{-}=-0.069$. We observe that cooperators slowly invade the population in panel (a) for $\alpha=-\beta$, and defectors take over the whole domain in panel (b) for $\alpha=1, \beta=0.1$ and $w=1$ for which the minimal wave speed is also $c_{0}^{-}=-0.069$. Figure 4 therefore shows that the spreading speed $c_{*}^{+}$( or $c_{*}^{-}$) of defectors increases (decreases) in $\alpha$. It can also be deducted from this fact that the difference $c_{*}^{+}-c_{*}^{-}$also increases in $\alpha$.

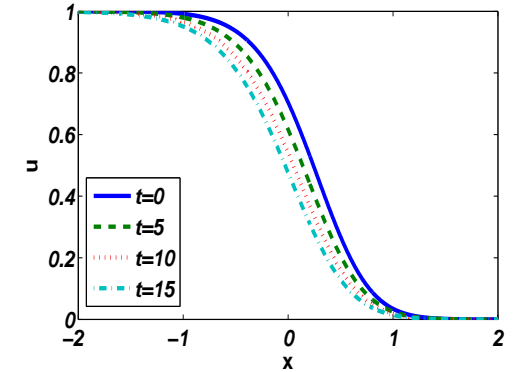

(a) Cooperators survive for $\alpha=-\beta=1$ and $w=0.1$.

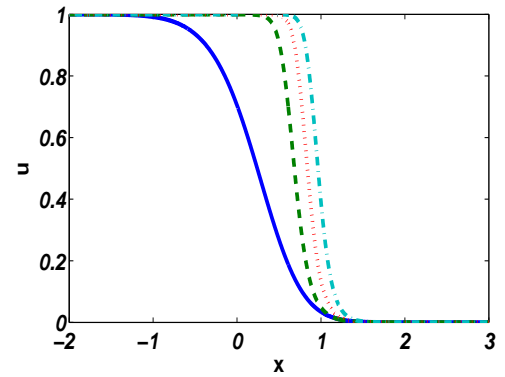

(b) Spread of defectors for $\alpha=1, \beta=-0.1$ and $w=1$.

Figure 4: Panels (a) and (b) display the effect of $\alpha$ on the preservation of cooperation in PD games. Successive waves are separated by 5 time units; for simulations, $\Delta x=0.002$ on $[-20,20]$ and $\Delta t=0.005$.

\subsection{Other evolutionary spatial games}

In this section we discuss the remaining spatial evolutionary games. Our aim is to illustrate the effect of spreading speeds (or traveling wave speeds) for SD and coordination games using simulations.

Figure 5 provides a numerical simulation of three cases given by (3.16), for which the underlying evolutionary spatial SD game has $(\alpha, \beta)=(-0.212,-1)$ and $w=0.792$. Kernel $J$ is centered Gaussian with $\sigma=1$, so that $c_{1}^{-}=-0.936$ and $c_{0}^{+}=2.743$. By Theorem 5 , an evolutionary spatial SD game with $c=5,1$ or -2 has strategy 1 , strategy 2 , and coexistence of strategies 1 and 2 as the evolutionary outcome of the game, respectively.

Panel (a) in Figure 5 illustrates that players using strategy 2 drives out individuals playing strategy 1 for $c=5$. Here one can easily notices that above the interior equlibria $e$ defectors vanish faster due to the difference between $c=5$ and minimal speeds of two equations (3.1) and (3.10). Panel (b) shows the coexistence of two types for $c=2$. Notice that the spreading speed is between two minimal speeds $c_{1}^{-}$and $c_{0}^{+}$. Thus above interior equlibrium $e$, players with strategy 1 drives out individuals playing strategy 2 . On 
the other hand below $e$, players with strategy 2 win. Lastly, panel (c) shows that players with strategy 1 take over the whole space for $c=-2$. Similarly, one can easily observe that speed of spread is faster below the equilibrium $e$.

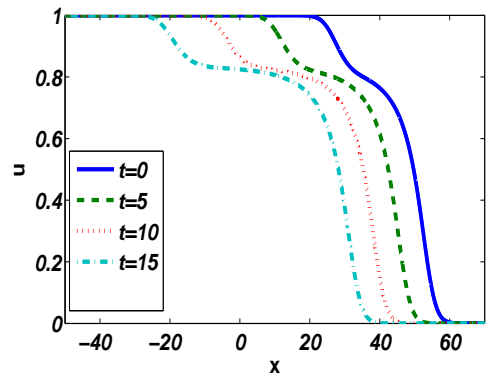

(a) Strategy 2 take over the population for $c=5$.

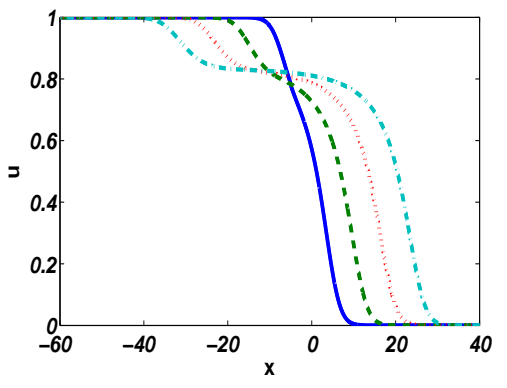

(b) Strategies 1 and 2 coexist for $c=1$

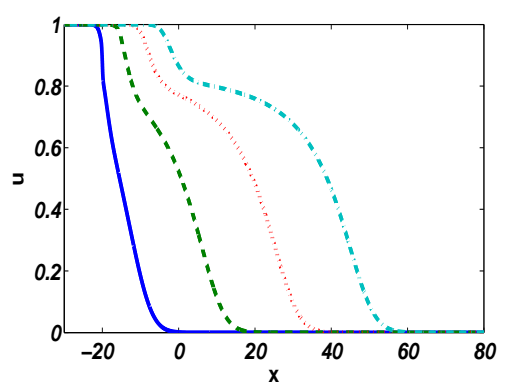

(c) Strategy 1 take over the population for $c=-2$.

Figure 5: Asymptotic behaviors of evolutionary spatial SD games. Successive curves are separated by 5 time units, $\Delta x=0.002$ on $[-100,100]$ and $\Delta t=0.0005$.

Coordination games are often employed in social sciences and economics to understand and solve coordination problems. In Figure 6, the underlying evolutionary spatial coordination game has $(\alpha, \beta)=(0.25,1)$ and $w=0.32$. Kernel $J$ is centered Gaussian with $\sigma=1$. For traveling wave speed $c=1$, defection sustains; while for $c=-1$, cooperation becomes dominant.

Similar results are reported by [21], where the evolutionary game is modeled by a reaction diffusion equation modeling mobile individuals in one dimensional space. Pareto dominant equlibrium in spatial coordination games determined by the sign of traveling wave speed $c$ since we assume that the interaction kernel is symmetric. In the case of asymmetric interactions, this result is subject to change. In this case, a theoretical bound on the traveling wave speed $c$ is given by [9].

\section{Selections of strains of an RNA virus: an application of ESG}

\subsection{Evolutionary spatial PD game for preserving cooperator strains}

In [54], an analysis was reported on modeling coinfection of a bacterial strain, P. phaseolicola by these two genotypes of viruses, which differ from each other by the multiplicity of infections (MOI). One strain 

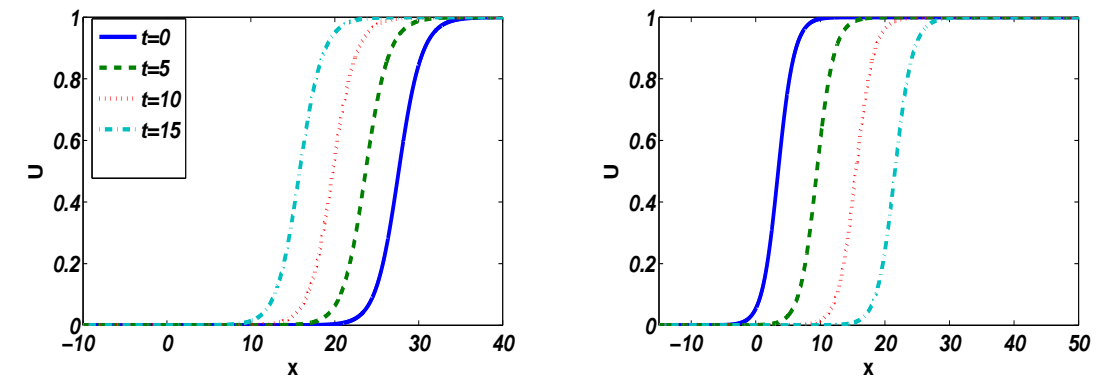

(a) Strategy 1 takes over the population for (b) Strategy 2 takes over the population for $c=1 \quad c=-1$

Figure 6: Asymptotic behaviors of evolutionary spatial coordination games. Successive curves are separated by 5 time units, $\Delta x=0.002$ on $[-100,100]$ and $\Delta t=0.0005$.

of the virus has high multiplicity with $\mathrm{MOI}=5$ and the other has low-multiplicity with $\mathrm{MOI}=0.002$ only. The former strain has a greater replication rate than the later strain. The mutant with high MOI is called the defector. Experiments show that the fitness of defectors increases as their initial frequency decreases. Second, they experimentally observed that defection is the only evolutionary outcome of this interaction in well mixed populations. These results imply that it is appropriate to model the interactions between the two strains of this RNA virus by a PD game.

The payoff matrix of this PD game was estimated by [54] from data as

$$
\mathbf{M}_{\text {virus }}=\left[\begin{array}{cc}
\mathrm{D} & \mathrm{C} \\
0.83 & 1.99 \\
0.65 & 1
\end{array}\right] \stackrel{D}{C} .
$$

It is easy to see from $\mathbf{M}_{\text {virus }}$ that defection is the only ESS for a spatial homogeneous population. Using the traditional unstructured replicator equation without spatial heterogeneity, Figure 7 displays the game dynamics in which defectors can take over the population even starting with a small initial frequency.

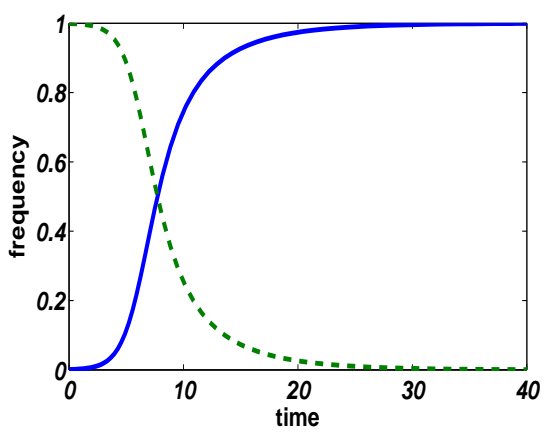

Figure 7: Solutions to the unstructured replicator equation with fitness matrix $\mathbf{M}_{\mathrm{virus}}$. The initial frequency of defectors is 0.001. Blue solid curve and green dashed curves are the frequencies of defectors and cooperators in time, respectively.

To refine the PD game with nonlocal spatial interactions, we let $\alpha=\left(m_{11}-m_{12}\right) / m_{\max }$ and $\beta=$ $\left(m_{22}-m_{21}\right) / m_{\max }$, where $m_{\max }=0.99$ is the maximal payoff difference in $\mathbf{M}_{\text {virus. }}$. The payoff matrix is 
therefore equivalent to

$$
\widetilde{\mathbf{M}}_{\text {virus }}=\left[\begin{array}{ccc}
\mathrm{D} & \mathrm{C} \\
0.181 & 0 \\
0 & -1
\end{array}\right] \quad \begin{gathered}
D \\
C
\end{gathered} .
$$

It is easy to see that $\widetilde{\mathbf{M}}_{\text {virus }}$ guarantee the linear determinancy of (3.1) as $\alpha+\beta<0$, i.e. Condition (P2) holds. In addition, for natural selection parameters $w<0.5497$, the quasi-monotonicity condition is satisfied as $w(-2 \beta-\alpha)<1$. We adopt the centered Gaussian kernel to model the nonlocal interactions. Simulation studies are displayed in Figure 1 in Section 4.1. Panel (a) in Figure 1 shows a transition of the evolutionary outcome of the game from defection to cooperation whenever the travelling wave speed is smaller than the minimal wave speed $c_{0}^{-}$. Panel (b) in Figure 1, on the other hand, displays the effect of the strength of nonlocal spatial interactions.

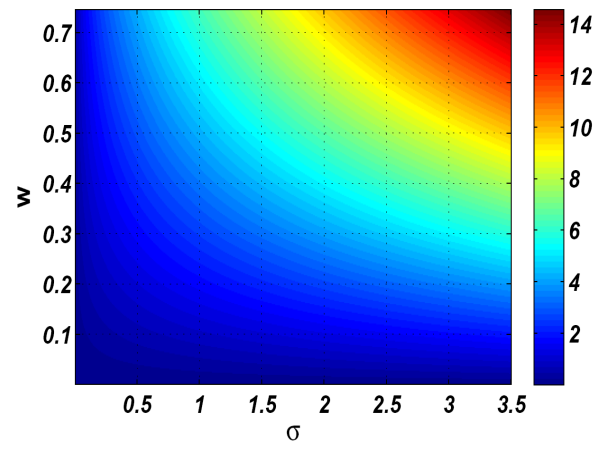

Figure 8: Effects of natural selection parameter $w$ and kernel parameter $\sigma$ on $c_{0}^{+}-c_{0}^{-}$.

As did in Section 3.2, the heat map in Figure 8 displays the effects of $\sigma$ and $w$ on $c_{0}^{+}-c_{0}^{-}$. We notice that for fixed natural selection parameter $w$, there always exists $\sigma_{w}$ such that $c_{0}^{+}-c_{0}^{-}$is sufficiently small for cooperation to be favored in this evolutionary spatial PD game. Nonlocal spatial interactions in model (3.1) therefore assist the RNA virus $\Phi H 2$ to sustain.

\subsection{An alternative approach}

To incoporate the spatial heterogeneity, one may model the frequency of defectors at $t \in \mathbb{R}^{+}$and $x \in \mathbb{R}$ by $r(x, t)$ satisfying

$$
\frac{\partial r}{\partial t}=d \frac{\partial^{2} r}{\partial x^{2}}+f(r)
$$

where the first term on the right hand side models spatial motility of the defectors and $f(r)$ is the replicator term modeling game dynamics. This spatial game model has been studied widely in the context of the reaction-diffusion equations, see [21] for example. For traveling wave solutions to (5.1), i.e. $r(x, t)=R(x+c t)$, it holds

$$
c R^{\prime}=d R^{\prime \prime}+f(R) .
$$

For $f(R)$ given in (3.17), the asymptotic behaviour of (5.2) is summarized as following.

Proposition 3. [18] For the spatial PD game modeled by (5.2), there exists a minimal front velocity $c_{\mathrm{PDE}}>0$. For any velocity $c>c_{\mathrm{PDE}}$ there exists a unique front connnecting two equilibria 0 and 1 . In addition if $\alpha<0$ the minimal velocity is given by $c_{\mathrm{PDE}}=2 \sqrt{-d \beta}$.

This result implies that, depending on the wave speed, cooperation can preserve and populate within a population of defection. One can easily observe that this PDE admits traveling wave solutions given 


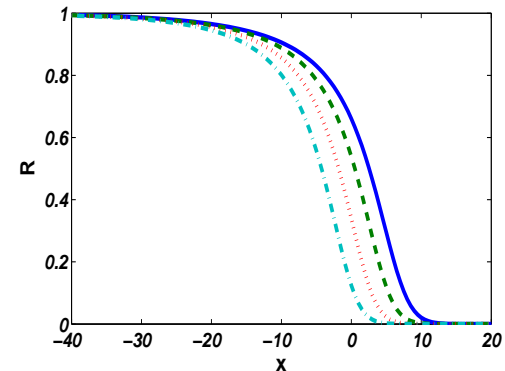

(a) Traveling wave solutions for $c=3.5$

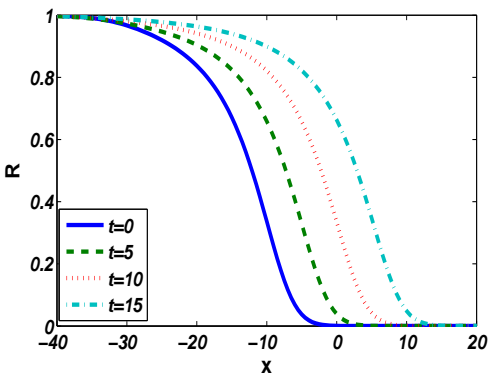

(b) Non-existence of traveling wave solutions for $c=1$

Figure 9: Panels (a) and (b) display the existence and non-existence of traveling waves of a spatial PD game modeled by (5.1).

$c \notin\left(-c_{\mathrm{PDE}}, c_{\mathrm{PDE}}\right)$. For simulations, we choose payoff matrix $\widetilde{\mathbf{M}}_{\mathrm{virus}}$. One can immediately obtain the minimal wave speed as $c_{\mathrm{PDE}}=1.718$ for diffusion rate $d=0.927$. Existence and non-existence of traveling waves are illustrated in Figure 9. Panel (a) in Figure 9 shows the formation of a traveling wave whose front towards the negative direction as time evolves, and panel (b) suggests that no traveling waves along the negative direction are formed and the solution converges to constant 1 on the domain. Therefore, cooperation sustains in panel (a) in Figure 9.

PDE model v.s. Non-local interaction model: Proposition 3 implies that defection is globally asymptotically stable for (5.1), while it is also globally asymptotically stable for (3.1) only if $0 \in\left(c_{*}^{-}, c_{*}^{+}\right)$by Theorem 2. Our evolutionary non-local spatial PD game in (3.1) is therefore essentially different from the spatial PD game in (5.2): if the nonlocal interaction kernel in (3.1) is skewed such that $0 \notin\left(c_{*}^{-}, c_{*}^{+}\right)$, then a small population of defectors cannot invade the cooperators. For example, consider the payoff matrix $\widetilde{\mathbf{M}}_{\mathrm{virus}}$. $c_{0}^{-}=0.0512$ and $c_{0}^{+}=1.377$ for the Gaussian kernel $J$ with mean $\mu=0.6$, standard deviation $\sigma=1$ and selection parameter $w=0.1$. Figure 10 illustrates solution to (3.1). It is obvious that cooperators take over the whole space with these parameters. Therefore it provides a demonstration of the effect of asymmetric interactions on global asymptotic stability of the nonlocal replicator equation (3.1).

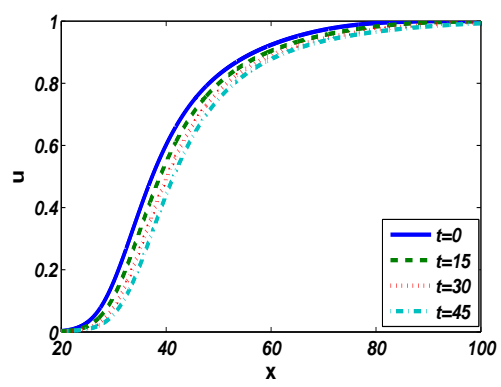

Figure 10: Invasion of cooperators under the nonlocal replicator dynamics (3.1) for asymmetric Gaussian kernel function with $\mu=0.6$ and $\sigma=1$, selection parameter $w=0.1$ and payof matrix $\widetilde{\mathbf{M}}_{\text {virus }}$. 


\section{Conclusions}

In this paper, from a stochastic process, we derive an integro-differential replicator equation to study evolutionary games in presence of nonlocal spatial interactions. In the microscopic model, individuals collect their payoffs based on a rule modeled by a discrete probability kernel and update their strategies accordingly. The derived integro-differential replicator equation can be viewed as the deterministic counterpart of spatial stochastic processes on microscopic scales. By considering the nonlocal interactions, this model is biologically more realistic than some traditional treatments, such as the homogeneous replicator equation.

We analyze the derived deterministic model for a variety of two-strategy games on an unbounded region. The necessary conditions for quasi-monotonicity and linear determinacy, which provide both mathematical conveniences and biological intuitions, are given and discussed in terms of model parameters. For example, it has been shown that the model is always quasi-monotone for small natural selection parameter. We focus on studying minimal traveling wave speed and/or the asymptotic speed of propagation, and our main results can be summarized as follows.

1. We investigate the minimal traveling wave speed (or the spreading speed) as a function of the variability of the nonlocal interaction kernel $\left.M^{\prime \prime}(s)\right|_{s=0}$, the payoffs, and the natural selection intensity. As a result, we found general minimal traveling wave speeds to preserve cooperation in PD games. Interestingly, we observe that cooperation wins for an evolutionary spatial PD game under a weak natural selection condition (P1).

2. A variety of kernels are studied numerically and compared in terms of how do they preserve cooperation in an evolutionary spatial PD game. We also apply the results to a PD game played by two strains of a RNA virus [54], and explore the effect of the nonlocal spatial interaction on the game dynamics for this real biological problem.

3. Spreading speeds, by which we can relax the linear determinacy condition, are studied and compared with minimal traveling waves speeds for evolutionary spatial PD games. We show that the spreading speed are equal to the minimal traveling wave speed under certain growth conditions. Therefore, the spreading speed can be characterized as the lowest speed of a class of traveling wave solutions whenever such a growth condition is satisfied.

4. We also study other types of evolutionary spatial games, including the SD, coordination and harmonic games. For the evolutionary spatial SD games, depending on the minimal traveling wave speed, three different scenarios separate the solution space. That is either strategy 1 or 2 take over the whole space, or they coexist. On the other hand, the derived model becomes bistable for an evolutionary spatial coordination games. That is, either strategy 1 or 2 may be favored depending on the traveling wave speed.

Finally, there are several interesting issues should be further explored or extended.

- In this paper, we focus on two-strategy games. The derived results may be extended naturally to $n$-strategy games with $n \geq 3$, such as the Rock-Scissors-Paper game. To study the traveling front in systems of integro-differential equations, results by [34] would be helpful.

- In this study, we consider the so-called biological replicator equations with linear fitness functions. It is interesting to generalize the linear fitness function to nonlinear forms, see for example [29, 26]. Evolutionary (nonlocal) spatial nonlinear games may have similar but more fruitful behaviors, particularly for the preservation of cooperation. 
- Numerically and biologically, it would be really important to estimate the model parameters, including the payoff matrix, the natural selection parameter and the interaction kernels from data. Notice that the interaction kernel is essentially a probability measure, the inverse problem for estimating the kernel would fall into the regime of nonparametric Bayesian or smoothing estimation scheme. We would like to leave this as a future work.

\section{Acknowledgments}

The authors are grateful to Professor Zhijun Wu for his generous supports. W.Z.'s research is partially supported by NSF-IIS (1545994); Y.K.'research is partially supported by NSF-DMS (1313312); NSFIOS/DMS (1558127) and The James S. McDonnell Foundation 21st Century Science Initiative in Studying Complex Systems Scholar Award (UHC Scholar Award 220020472).

\section{Appendix A Technical Proofs}

\section{A.1 Mesoscopic limits}

Denote $S=\{1,2, \cdots, n\}$ the set of strategies. The evolution of the system is given by the continuous time process $\left\{\theta_{t}\right\}$ with the state space $S^{\Lambda}$. We denote the configuration of the lattice by $\theta:=\theta_{t}$ at time $t$. Each individual updates the strategy, independently of all others, in response to an exponential clock with rate 1 and changes the strategy according to $r(\dot{x}, \theta, k)$.

Definition A.1. The empirical measure $\pi^{\gamma}: S^{A^{\gamma}} \rightarrow \mathbb{P}(A \times S)$ is given by

$$
\pi^{\gamma}(\theta)=\frac{1}{\left|A^{\gamma}\right|} \sum_{\dot{x} \in A^{\gamma}} \delta_{(\gamma \dot{x}, \theta(\dot{x}))}
$$

where $\mathbb{P}(A \times S)$ denotes the set of all probability measures on $A \times S$.

Let $\theta_{t}^{\gamma}$ be the stochastic process with generator

$$
\left(L^{\gamma} g\right)(\theta)=\sum_{\dot{x} \in A^{\gamma}} \sum_{k \in S} r^{\gamma}(\dot{x}, \theta, k)\left(g\left(\theta^{\dot{x}, k}\right)-g(\theta)\right)
$$

We make following assumptions on strategy revision rate $r^{\gamma}(\dot{x}, \theta, k)$ : there exists $r(x, i, k, \pi) \in \mathbb{R}$ for $x \in A$, $i, k \in S$ and $\pi \in \mathbb{P}(A \times S)$ such that

(R1) $\lim _{\gamma \rightarrow 0} \sup _{\dot{x} \in A^{\gamma}, \theta, k}\left|r^{\gamma}(\dot{x}, \theta, k)-r\left(\gamma \dot{x}, \theta(x), k, \pi^{\gamma}(\theta)\right)\right|=0$,

(R2) $r(x, i, k, \pi)$ is uniformly bounded, that is there exists $M>0$ such that

$$
\sup _{x \in A, i, k}|r(x, i, k, \pi)| \leq M, \text { and }
$$

(R3) $r(x, i, k, f m)$ is Lipschitz in $x$, i.e. $\sup _{x \in A, i, k}\left|r\left(x, i, k, u_{1} m\right)-r\left(x, i, k, u_{2} m\right)\right| \leq L|| u_{1}-u_{2} \|_{L^{1}}$, where $m=$ $d x \otimes d i$ with Lebesgue measure $d x$ on $A$ and counting measure $d i$ on $S$.

The next result connects the stochastic process with the integro-differential equation. 
Theorem A.1. ([29]) Assume Conditions (R1)-(R3) are satisfied. Let $\boldsymbol{u} \in L^{\infty}(A \times S)$ satisfy $0 \leq u_{i} \leq 1$ and $\sum_{i \in S} u_{i}=1$ for all $x \in A$. In addition, assume that the initial distribution is a family of measures with slowly varying parameter associated to the profile of $\boldsymbol{u}$. Then for every $T>0$, as $\gamma \rightarrow 0$

$$
\pi_{t}^{\gamma}\left(\theta_{t}\right) \rightarrow \boldsymbol{u}(t, x) m
$$

in probability uniformly for $0 \leq t \leq T$; and for $x \in A, i \in S, \boldsymbol{u}$ satisfies

$$
\frac{\partial u_{i}}{\partial t}(x, t)=\sum_{k \in S} r(x, k, i, \boldsymbol{u}) u_{k}(x, t)-u_{i}(x, t) \sum_{k \in S} r(x, i, k, \boldsymbol{u})
$$

with initial condition $\boldsymbol{u}(x, 0)$.

The corollary below can be obtained from the above theorem. We refer to [5] for the proof.

Corollary A.1. Suppose that the interaction is spatially homogeneous with the same assumptions on rate $r$. Assume that there exists $\boldsymbol{\rho}_{0} \in \Delta$ such that the initial condition satisfies $\boldsymbol{\eta}_{0}^{N} \rightarrow \boldsymbol{\rho}_{0}$ in probability as $N \rightarrow \infty$, then for every $T>0$

$$
\boldsymbol{\eta}_{t}^{N} \rightarrow \boldsymbol{\rho}
$$

in probability uniformly for $0 \leq t \leq T$ as $N \rightarrow \infty$ and $\boldsymbol{\rho}$ satisfies

$$
\left\{\begin{array}{l}
\frac{d \rho_{i}}{d t}=\sum_{k \in S} r(k, i, \boldsymbol{\rho}) \rho_{k}-\rho_{i} \sum_{k \in S} r(i, k, \boldsymbol{\rho}) \\
\rho_{i}(0)=\rho_{0 i}
\end{array}\right.
$$

where $r(k, i, \boldsymbol{\rho})=\rho_{i} F\left(\sum_{l \in S} m_{i l} \rho_{l}-\sum_{l \in S} m_{k l} \rho_{l}\right)$ (for $F$ discussed in Section 2.2.1).

\section{A.1.1 Replicator rule and replicator equations}

The rate function (2.3) with globally Lipschitz continuous $F(\cdot)$ satisfies Conditions (R1)-(R3) [28]. Theorem A.1 therefore gives the replicator equations for $F(s)=1+s / m_{\max }$. The corresponding $r(x, i, k, \boldsymbol{u})$ is given by (2.7).

Corollary A.2. Without the spatial heterogeneity, rate function (2.3) with $F(s)=1+w s / m_{\max }$ gives the replicator equations.

Proof. Without the spatial heterogeneity, the rate function can be written as

$$
r(i, k, \boldsymbol{\rho})=\rho_{k}\left\{1+w \sum_{l \in S}\left(m_{k l}-m_{i l}\right) \rho_{l}\right\}
$$

by taking $\boldsymbol{u}(x, t)=\rho$. By Corollary A.1, we have

$$
\begin{aligned}
\frac{d \rho_{i}}{d t} & =\sum_{k \in S} r(k, i, \rho) \rho_{k}-\rho_{i} \sum_{k \in S} r(i, k, \rho) \\
& =\sum_{k \in S} \rho_{i}\left\{1+w \sum_{l \in S}\left(m_{i l}-m_{k l}\right) \rho_{l}\right\} \rho_{k}-\rho_{i} \sum_{k \in S} \rho_{k}\left\{1+w \sum_{l \in S}\left(m_{k l}-m_{i l}\right) \rho_{l}\right\} \\
& =\sum_{k \in S}\left[\rho_{i}\left\{1-w \sum_{l \in S}\left(m_{i l}-m_{k l}\right) \rho_{l}\right\} \rho_{k}-\rho_{i} \rho_{k}\left\{1+w \sum_{l \in S}\left(m_{k l}-m_{i l}\right) \rho_{l}\right\}\right]
\end{aligned}
$$




$$
\begin{aligned}
& =2 w \rho_{i} \sum_{k, l \in S}\left(m_{i l}-m_{k l}\right) \rho_{k} \rho_{l} \\
& =2 w\left(\rho_{i} \sum_{l \in S} m_{i l} \rho_{l}-\rho_{i} \sum_{k, l \in S} m_{k l} \rho_{l} \rho_{k}\right),
\end{aligned}
$$

which gives the replicator equations by changing the time scale.

Now we obtain the nonlocal replicator equations using Theorem A.1 and (2.7).

$$
\begin{aligned}
\frac{\partial u_{i}}{\partial t} & =\sum_{k \in S} r(x, k, i, \boldsymbol{u}) u_{k}(x, t)-u_{i}(x, t) \sum_{k \in S} r(x, i, k, \boldsymbol{u}) \\
& =\sum_{k \in S} J * u_{i}\left[1+w J *\left\{\sum_{l \in S}\left(m_{i l}-m_{k l}\right) u_{l}\right\}\right] u_{k}-u_{i} \sum_{k \in S}\left(J * u_{k}\right)\left[1-w J *\left\{\sum_{l \in S}\left(m_{i l}-m_{k l}\right) u_{l}\right\}\right] \\
& =\sum_{k \in S}\left[\left\{u_{k}\left(J * u_{i}\right)-u_{i}\left(J * u_{k}\right)\right\}-w\left\{u_{k}\left(J * u_{i}\right)+u_{i}\left(J * u_{k}\right)\right\} \cdot\left\{\sum_{l \in S}\left(m_{k l}-m_{i l}\right)\left(J * u_{l}\right)\right\}\right] .
\end{aligned}
$$

As $\sum_{k \in S} u_{k}=\sum_{k \in S} J * u_{k}=1$, we have

$$
\frac{\partial u_{i}}{\partial t}(x, t)=\left(J * u_{i}\right)-u_{i}+w\left[\left\{\left(J * u_{i}\right)+u_{i}\right\} \sum_{l \in S} m_{i l}\left(J * u_{l}\right)-\sum_{k, l \in S} m_{k l}\left\{u_{k}\left(J * u_{i}\right)+u_{i}\left(J * u_{k}\right)\right\}\left(J * u_{l}\right)\right] .
$$

Without spatial structures, by taking $u_{i}(x) \equiv \rho_{i}$ we get $J * u_{i}=\rho_{i}$ so that the above equation reduces to the replicator equation.

\section{A.2 Quasi-monotonicity Condition (P1)}

As defined in Section 3.1, equation (3.1) is quasi-monotone if its right hand side is increasing in $J * u$. Denote the right hand side of (3.1) by

$$
f(u, v)=v-u+w(u+v-2 u v)\{(\alpha+\beta) v-\beta\}
$$

where $(u, v) \in \Omega=\{[0,1] \times[0,1] \mid v \leq u\}$ by Young's inequality. It is readily to see that

$$
g(u, v):=f_{v}(u, v)=1+w\{(\alpha+3 \beta) u+2(\alpha+\beta) v-4(\alpha+\beta) u v-\beta\},
$$

which is required to be positive for all $(u, v) \in \Omega, \alpha \in[0,1], \beta \in[-1,0]$ and $w \in[0,1)$ to guarantee the quasi-monotonicity of (3.1). It is easy to see that the stationary point of $g$ is a saddle point as the Hessian $\nabla^{2} g$ is indefinite. Therefore, the minimum value of $g$ is achieved on the boundary $\partial \Omega$. Since $g$ is quadratic, it is enough to study the corners of $\Omega$ where

$$
\begin{aligned}
& g(0,0)=1-w \beta \\
& g(1,0)=1+w(\alpha+2 \beta), \\
& g(1,1)=1-w \alpha .
\end{aligned}
$$

Hence, $g(u, v) \geq \min \{1-w \beta, 1-w \alpha, 1+w(\alpha+2 \beta)\}$ on $\Omega$. Both $1-w \beta$ and $1-w \alpha$ are positive for $\alpha \in[0,1], \beta \in[-1,0]$ and $w \in[0,1)$. Hence, $g(u, v)>0$ whenever $1+w(\alpha+2 \beta)>0$. That is, the right hand side of (3.1) is increasing in $J * u$ whenever $1+w(\alpha+2 \beta)>0$, which justifies Condition (P1) in Section 3.2. 


\section{A.3 Existence and uniqueness of solutions to (3.7) and comparison principle}

The following theorem provides existence, uniqueness and monotonicity of the solution to (3.7) in the space of bounded uniformly continuous functions $B U C\left(\mathbb{R} \times \mathbb{R}^{+}\right)$.

Theorem A.2. Assume Conditions (J1)-(J3) are satisfied. Then there exists a unique solution $u(x, t) \in$ $B U C\left(\mathbb{R} \times \mathbb{R}^{+}\right)$to (3.7) with an initial condition $0 \leq u(x, 0) \leq 1$ such that $0 \leq u \leq 1$. Moreover, suppose that Condition (P1) holds and for $i=1,2$ and $u_{i}$ is the solution to (3.7) corresponding the continuous initial datum $0 \leq u_{i}(x, 0)=u_{0 i} \leq 1$.

$$
\text { If } 0 \leq u_{01} \leq u_{02} \leq 1 \text { then } 0 \leq u_{1} \leq u_{2} \leq 1
$$

for all $(x, t) \in \mathbb{R} \times \mathbb{R}^{+}$.

The proof of this theorem follows a standard method in [48] that we recall for the sake of completeness.

Proof. We work on the space of bounded uniformly continuous functions $X_{T}=B U C(\mathbb{R} \times[0, T])$ and $X_{\infty}=$ $B U C\left(\mathbb{R} \times \mathbb{R}_{+}\right)$.

Step 1. For $v \in X_{\infty}$ such that $0 \leq v \leq 1$, let $u$ be a solution to equation

$$
\frac{\partial}{\partial t} u=(J * v)-u+w(u+(J * v)-2 u(J * v))((\alpha+\beta)(J * v)-\beta)
$$

with the initial data $u=u_{0}$ on $\mathbb{R} \times\{0\}$. By assumptions on $J$ and $v, 0 \leq J * v \leq 1$. Notice that $\partial u / \partial t=(J * v)[1+w((\alpha+\beta)(J * v)-\beta)] \geq 0$ if $u=0$ at some point $(x, t)$. The trajectory of $u$ is therefore always beyond 0 . Similarly, $\partial u / \partial t \leq 0$ if $u=1$ at some point $(x, t)$. Hence, $u \in[0,1]$.

To show the existence and uniqueness of solutions to (A.3), we define the operator $P[u]: C(\mathbb{R} \times$ $\left.\mathbb{R}_{+},[0,1]\right) \rightarrow C\left(\mathbb{R} \times \mathbb{R}_{+}, \mathbb{R}\right)$ by

$$
P[u](x, t):=(J * v)+a u+w(u+(J * v)-2 u(J * v))((\alpha+\beta)(J * v)-\beta) .
$$

Operator $P[u]$ is non-decreasing for sufficiently large $a>0$ with respect to pointwise ordering. Hence, (A.3) can be written as

$$
\frac{\partial}{\partial t} u(x, t)=-(1+a) u(x, t)+P[u](x, t),
$$

which is further written as

$$
u(x, t)=G[u](x, t):=\phi(x) e^{-(1+a) t}+\int_{0}^{t} e^{-(1+a)(t-s)} P[u](x, s) d s .
$$

As $0 \leq v(t, x), J * v(t, x) \leq 1$, it is easy to see that $\left|P\left[u_{1}\right]-P\left[u_{2}\right]\right| \leq(a+3 w)\left|u_{1}-u_{2}\right|$. Consider $X_{\infty}$ equipped with the norm $\|v\|_{\lambda}=\sup _{(x, t)}|v(x, t)| e^{-\lambda t}$, we have

$$
\left|G\left[u_{1}\right]-G\left[u_{2}\right]\right| e^{-\lambda t} \leq(a+3 w) \int_{0}^{t} e^{-(1+a+\lambda)(t-s)} e^{-\lambda s}\left|u_{1}-u_{2}\right| d s .
$$

Therefore,

$$
\left\|G\left[u_{1}\right]-G\left[u_{2}\right]\right\|_{\lambda} \leq(a+3 w) \frac{1-e^{-(1+a+\lambda) t}}{1+a+\lambda}\left\|u_{1}-u_{2}\right\|_{\lambda} \leq \frac{a+3 w}{1+a+\lambda}\left\|u_{1}-u_{2}\right\|_{\lambda} .
$$

Choose $\lambda$ large enough so that $\frac{a+3 w}{1+a+\lambda}<1$ and mapping $G$ is therefore a contraction. By Banach fixed point theorem, there exists a unique solution to (A.3). 
Step 2. Hereafter, we consider the space $X_{T}$ equipped with the norm $\|v\|_{T}=\sup _{(x, t)}|v(x, t)|$. We will show that mapping $v \rightarrow u$ is a contraction. Let $v_{i} \in X_{T}(i=1,2)$ and $u_{i}$ denote the corresponding solutions to equation (A.3) with the same initial data. Let $u=u_{1}-u_{2}$ and $v=v_{1}-v_{2}$, so that

$$
\frac{\partial}{\partial t} u=\Upsilon_{1} u+\Upsilon_{2} J * v,
$$

where $\Upsilon_{1}=-\left[1-\beta w-w(\alpha+3 \beta)\left(J * v_{1}\right)+2(\alpha+\beta) w\left(J * v_{1}\right)^{2}\right]$ and $\Upsilon_{2}=\left[1-\beta w+w(\alpha+3 \beta) u_{2}+\right.$ $\left.(\alpha+\beta) w\left(J *\left(v_{1}+v_{2}\right)\right)\left(1-2 u_{2}\right)\right]$. Integration up to time $t$ yields

$$
u=\int_{0}^{t} \Upsilon_{1} u d s+\int_{0}^{t} \Upsilon_{2} J * v d s
$$

which implies

$$
|u| \leq \int_{0}^{t}\left|\Upsilon_{1}\right||u| d s+\int_{0}^{t}\left|\Upsilon_{2}\right||J * v| d s .
$$

Let $K_{k}=\max _{u_{i}, v_{j} \in[0,1]} \Upsilon_{k}$ for $i, j, k=1,2$, and take the supremum over $(x, t) \in \mathbb{R} \times[0, T]$, we get

$$
\|u\|_{T} \leq \frac{T K_{2}}{1-T K_{1}}\|v\|_{T} .
$$

For sufficiently small $T, T K_{2} /\left(1-T K_{1}\right)<1$. Hence, for sufficiently small $T, v \rightarrow u$ is a contraction that preserves the closed subset $0 \leq v \leq 1$, and therefore admits a unique fixed point. Since $T$ is independent of the size of the data, this argument can be iterated from $T$ to any desired time interval.

Step 3. Let $u_{i}$ be the solutions to (3.7) corresponding to the initial datum $u_{0 i}$ satisfying the hypotheses of the theorem, set $u=u_{1}-u_{2}$. Then we get an equation of the form (A.4) with

$$
\Upsilon_{1}=-\left[1+\beta w-w(\alpha+3 \beta)\left(J * u_{1}\right)+2(\alpha+\beta) w\left(J * u_{1}\right)^{2}\right]
$$

and

$$
\Upsilon_{2}=\left[1-\beta w+w(\alpha+3 \beta) u_{2}+(\alpha+\beta) w\left(J *\left(u_{1}+u_{2}\right)\right)\left(1-2 u_{2}\right)\right] .
$$

Multiplying this equation by $\operatorname{sign}_{+}(u)$ and using the fact that $\Upsilon_{2}>0$ as (P1) holds, we get

$$
u_{+}(x, t) \leq \frac{t K_{2}}{1-t K_{1}} \int_{0}^{t} J * u_{+}(x, s) d s \leq \frac{t K_{2}}{1-t K_{1}}\left\|u_{+}\right\|_{t} .
$$

Using the same argument as above, for small $t$, we have $t K_{2} /\left(1-t K_{1}\right)<1$. It follows that $u_{+}=0$ in $[0, t]$ since $\left(u_{01}-u_{02}\right)_{+}=0$. This result can be extended to all time points by continuity. We therefore have the desired assertion (A.2) obtained.

\section{A.4 Proof of Theorem 1}

The following proposition provides the existence of monotone traveling waves connecting two equilibria of traveling wave equation (3.5).

Proposition A.1. Suppose $J$ satisfies (J1) and the quasi-monotonicity condition hold, then (3.5) admits traveling wave solutions for $c \geq c_{0}^{+}$and $c \leq c_{0}^{-}$for nonzero constants $c_{0}^{-}$and $c_{0}^{+}$. 
Proof. The existence of solutions to (3.5) for $c>c_{0}^{+}$was proved in [50]. For $c<c_{0}^{-}$, we can establish the same results by constructing appropriate sub and super solutions. We prove the existence of traveling waves for the minimal speed $c=c_{0}^{+}$following [6], to which the proof for the case $c=c_{0}^{-}$is analog.

Let $c_{n}>c_{0}$ and $c_{n} \rightarrow c_{0}$ as $n \rightarrow \infty$. Let $U_{n}$ denote the traveling wave solution to (3.5) with speed $c_{n}$. Thus, $0 \leq U_{n} \leq 1$ and the solution is uniformly bounded by the existence of solution. As $\int_{\mathbb{R}} J(y) d y=1$, $J * U$ is uniformly bounded by 1 . Hence,

$$
\left|U_{n}^{\prime}\right| \leq c_{n}^{-1}\left(\left|J * U_{n}\right|+\left|U_{n}\right|-w \beta\left|U_{n}+J * U_{n}\right|\right) \leq 4 c_{n}^{-1} .
$$

Hence, $U_{n}^{\prime}$ is also uniformly bounded.

We have $\left|U_{n}(s)-U_{n}(t)\right|=|s-t|\left|U_{n}^{\prime}(\nu)\right|$ for some $\nu$ between $s$ and $t$. By uniform boundedness of $U_{n}^{\prime}$, it follows that $U_{n}$ is equicontinuous for all $n$. Hence, the equicontinuity of $U_{n}$ implies that for any $\epsilon>0$ there exists $\delta>0$ such that

$$
\left|J * U_{n}(s)-J * U_{n}(t)\right| \leq \int_{\mathbb{R}}\left|U_{n}(s+y)-U_{n}(t+y)\right| J(y) d y \leq \epsilon
$$

for $|s-t|<\delta$. Likewise, we have the equicontinuity of $U_{n}^{\prime}$. Therefore, $U_{n}, J * U_{n}$, and $U_{n}^{\prime}$ are uniformly bounded and equicontinuous for all $n$.

By Arzela-Ascoli theorem, there exists a subsequence $\left\{U_{n_{k}}\right\} \subset\left\{U_{n}\right\}$ such that $J * U_{n_{k}}$ and $U_{n_{k}}^{\prime}$ converges uniformly on every bounded interval. Denote the limit of $U_{n_{k}}$ by $U$, then $U$ is differentiable and $U_{n_{k}}^{\prime} \rightarrow U^{\prime}$. Similarly, $J * U_{n_{k}} \rightarrow J * U$. Passage of limit in (3.5) yields

$$
-c_{0} U^{\prime}=(J * U)-U+w\{U+(J * U)-2 U(J * U)\}\{(\alpha+\beta)(J * U)-\beta\},
$$

as $n \rightarrow \infty$, which implies that $U$ is the solution to equation (3.5) with minimal speed $c_{0}$.

Theorem 2, which is proved in Section A.5 below, immediately yields the following corollary.

Corollary A.3. If $c \in\left(c_{0}^{-}, c_{0}^{+}\right)$, there are no non-constant solutions to (3.5).

Theorem 1 is then implied by Proposition A.1 and Corollary A.3.

\section{A.5 Outlines of the proof of Theorem 2}

Using the techniques developed by [32], we outline the proof of Theorem 2 in this section. For (3.7), there is a family of time- $t$ maps $Q_{t}$ defined by $Q_{t}\left[u_{0}\right]:=u(x, t)$. That is, $Q_{t}$ takes the initial values $u_{0}=u(\cdot, 0)$ to $u(x, t)$ at time point $t$. The semigroup property of the map naturally follows from the existence and uniqueness of the solution. It is noticed that, for fixed $t, Q_{t}$ satisfies the conditions in [32]. That is,

(i). $Q_{t}$ satisfies the order preserving property, which follows from the comparison principle given in A.2;

(ii). for a PD game, there are only two spatially homogeneous fixed points 0 and 1 and 1 is an asymptotically stable fixed point of $Q_{t}$ for any constant initial condition; also, by the comparison principle, $0 \leq$ $Q_{t}\left[u_{0}\right] \leq 1$ is continuous for any continuous $u_{0} \in[0,1]$;

(iii). $Q_{t}$ is translation invariant since $v(x, t)=u(x-y, t)$ is a solution to (3.7) whenever $u(x, t)$ is; and

(iv). $Q_{t}$ admits continuity, which is obtained by using techniques similar to [15, Lemma 2.2] and [57, Lemma 3.1]. 
Consider the time-1 map $Q:=Q_{1}$ and choose a continuous function $\phi$ such that $\phi$ is non-increasing in $x$, $\phi(x)=0$ for all $x \geq 0$, and $0 \ll \phi(-\infty) \ll 1$. We can therefore define the spreading speed using $\phi$ : let $a_{0}(c ; s)=\phi(s)$ and define $a_{n}(c ; s)$ by

$$
a_{n+1}(c ; s)=\max \left\{\phi(s), Q\left[a_{n}(c ; s)\right](s+c)\right\}
$$

The operator defined above is also order preserving. By definition, it is easy to see that $a_{1} \geq a_{0}=\phi(s)$. Then, $a_{n} \leq a_{n+1} \leq 1$ follows from inductions. Also, $a_{n}(c ; s)$ is non-increasing in $c$ and $s$. Hence, $a_{n}$ converges to some $a(c ; s)$, which is non-increasing in $c$ and $s$ and bounded by 1 . From the arguments in [34], $a(c ; \pm \infty)$ are equilibria of the time-1 map $Q$. In particular $a(c ; \infty)=1$ as noted in [32].

Consider another initial condition $\hat{\phi}$ satisfying the above properties of $\phi$. Then, there exists $\hat{a}_{n}(c ; s)$ whose limit is $\hat{a}(c ; \infty)$. As discussed in [32], one can show that $a_{N}(c ; x-\tau) \geq \hat{\phi}(x)$ with a translation $\tau$ for some $N>0$. By the comparison principle, it can be shown that $\hat{a}(c ; \infty)=a(c ; \infty)$. Hence, we can conclude that $a(c ; \infty)$ is independent of the choice of $\phi$. Therefore, we can define the slowest spreading speed by

$$
c_{+}^{*}=\sup \{c: a(c ; \infty)=1\} .
$$

By modifying the above discussion, one can also define the spreading speeds in the negative direction as follows:

$$
c_{-}^{*}=\inf \{c: a(c ;-\infty)=1\} .
$$

Hence, the result follows from [32, Theorem 2.2] for the time-1 map $Q_{1}$. Following the proof in [33, Theorem 2.17], we can also show that $c_{ \pm}^{*}$ are critical spreading speeds for continuous time semi-flow. Thus we have the desired assertion. Addtionally, the equality of $c_{*}^{+}$and $c_{0}^{+}$follows from [56, Theorem 3.4].

\section{A.6 Proof of Theorem 3}

We need to verify conditions (A1) (translation invariance), (A3) (point- $\alpha$-contraction), (A4) (orderpreserving), and (A5) (monostability) in [16] to use their Theorem 4.2 to show the existence of traveling wave solutions. In fact, conditions (A1), (A4) and (A5) are essentially conditions (i)-(iii) in Appendix A.5, which have been verified above. It remains to verify condition (A3), that is to show the solution operator $Q_{t}$ defined in Appendix A.5 satisfies the point- $\alpha$-contraction. Denote $M_{1}$ the set of bounded, continuous and non-increasing functions from $\mathbb{R}$ to $[0,1]$. For any bounded subset $\mathcal{U}_{1} \subset M_{1}$ the set $Q_{t}\left[\mathcal{U}_{1}\right](0)$ is bounded due to the uniform boundedness of the derivative $u_{t}$ for all $t$ and for any initial condition $\phi \in \mathcal{U}_{1}$. As any bounded subset of $\mathbb{R}$ is precompact, the point- $\alpha$ contraction property of $Q_{t}$ follows. Hence, by $[16$, Theorem 4.2 , we have the existence of traveling waves.

We further show the smoothness of the wave profiles. Consider operator $\mathcal{F}$ in (3.7). Equation (3.7) can be written into an integral equation

$$
v(x, t)=v_{0}(x) e^{-(1+\beta w) t}+\int_{0}^{t} e^{-(1+\beta w)(t-s)} \mathcal{F}(v,(J * v))(x, s) d s .
$$

Take $v(x, t)=W(x-c t)$, the traveling wave profile $W$ solves the following integral equation

$$
W(x-c t)=W(x) e^{-(1+\beta w) t}+\int_{0}^{t} e^{-(1+\beta w)(t-s)} \mathcal{F}(W,(J * W))(x-c s) d s .
$$

The right hand side of above equation is differentiable with respect to $t$. It follows that the wave profile is smooth and therefore a classical solution to (3.7). 


\section{A.7 Subsolution to Equation (3.13)}

Lemma A.1. Assume Conditions (J1)-(J3) and assumptions of Theorem 4 hold. Then, for any $\xi \in \mathbb{R}$, Let $u(x, 0)$ be an initial condition that is non-zero only in negative half plane and let $u$ be solution to the initial value problem (3.13). Then $\lim _{t \rightarrow \infty} u(\xi, t)$ is a constant solution to the equation other than 0 if $c \in\left(c_{0}^{-}, c_{0}^{+}\right)$ with $c_{0}^{ \pm}$defined by (3.3) and (3.4).

We prove Lemma A.1 using techniques developed by [2] and [35]. First, consider function

$$
\Delta_{c}(s):=-c s+d_{1} M(s)-d_{2}
$$

defined on $\mathbb{R}$ for $d_{1}=1-\beta w$ and $d_{2}=1+\beta w$. We have $\Delta_{c}^{\prime \prime}(s)=d_{1} \int_{\mathbb{R}} y^{2} e^{s y} J(y) d y$, which implies $\Delta_{c}(s)$ is convex on $\mathbb{R}$. By the continuity of $\Delta_{c}(s)$, there exist two unique points $s_{0}^{+}\left(d_{1}, d_{2}\right)$ and $s_{0}^{-}\left(d_{1}, d_{2}\right)$ such that $\Delta_{c}\left(s_{0}^{ \pm}\left(d_{1}, d_{2}\right)\right)=0$ for any $c \notin\left[c_{0}^{-}, c_{0}^{+}\right]$. The result follows from the discussion in [50, p.64].

Consider function $A(s):=\left[d_{1} \int_{\mathbb{R}} J(y) e^{s y} d y-d_{2}\right] / s \in\left\{c: \Delta_{c}(s)=0\right\}$. Assumptions on $J$ implies that $A\left(s_{0}^{-}\left(d_{1}, d_{2}\right)\right)=\sup \left\{c: \Delta_{c}(s)=0, s<0\right\}$ and $A\left(s_{0}^{+}\left(d_{1}, d_{2}\right)\right)=\inf \left\{c: \Delta_{c}(s)=0, s>0\right\}$, so that $A\left(s_{0}^{ \pm}\right)=c_{0}^{ \pm}\left(d_{1}, d_{2}\right)$ by definitions.

\section{A.7.1 Classical results}

In this section we provide some classical results by $[2,35]$ concerning the spreading speeds of equation (3.13). Note also that the results are also given for equation (3.10) in moving frame.

Lemma A.2. Let $c \in\left(c_{0}^{-}, c_{0}^{+}\right)$. There exists a function $V(\xi)>0$ on $(0, \pi / \tau)$ such that $Q_{c}[\epsilon V(\xi)] \geq 0$ and

$$
Q_{c}[\epsilon V]>0 \text { on }(-\pi / \tau, 0)
$$

for sufficiently small $\epsilon, \tau>0$.

Proof. Take $V_{0}(\xi)=\exp (-s \xi) \sin (-\tau \xi)$, where $s \in S_{0}:=\left(s_{0}^{-}, s_{0}^{+}\right) \backslash\{0\}$. Consider the linear equation

$$
\frac{\partial u}{\partial t}:=L_{c}[u]=-c u_{\xi}+d_{1} J * u-d_{2} u .
$$

Here we can easily calculate $V_{\xi}$ and $J * V$ as follows:

$$
\begin{aligned}
V_{0 \xi} & =-s V_{0}-\exp (-s \xi) \cos (\tau \xi) \\
J * V_{0} & =\int_{\mathbb{R}} J(y) V_{0}(\xi-y), d y=\exp (-s \xi) \int_{\mathbb{R}} J(y) e^{s y} \sin (\xi+\tau y) .
\end{aligned}
$$

Then,

$$
L_{c}\left[V_{0}\right](\xi)=\left[-c s+d_{1} \int_{\mathbb{R}} J(y) e^{s y} \cos (\tau y) d y-d_{2}\right] V_{0}(\xi)+\left[-c \tau+d_{1} \int_{\mathbb{R}} J(y) e^{s y} \sin (\tau y) d y\right] e^{-s \xi} \cos (\tau \xi) .
$$

One can easily observe that $L_{c}[V]>0$ on $(-\pi / \tau, 0)$ if the following conditions are satisfied:

$$
\begin{aligned}
& \mathbb{A}(s, \tau):=\frac{1}{s}\left[d_{1} \int_{\mathbb{R}} J(y) e^{s y} \cos (\tau y) d y-d_{2}\right]>c \text { if } s>0 \\
& \mathbb{A}(s, \tau)<c \text { if } s<0 \\
& \mathbb{B}(s, \tau):=\frac{d_{1}}{\tau}\left[\int_{\mathbb{R}} J(y) e^{s y} \sin (\tau y) d y\right]=c .
\end{aligned}
$$


Denote the limits of $\mathbb{A}(s, \tau)$ and $\mathbb{B}(s, \tau)$ by $A(s)$ and $B(s)$ as $\tau \rightarrow 0$. By the assumptions on $J$, the

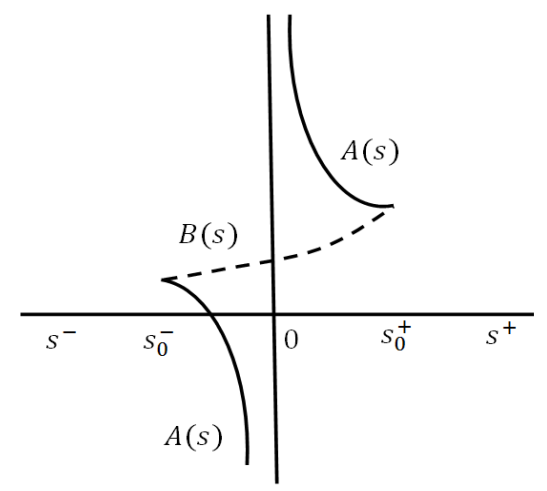

Figure A.1: Illustration of $A(s)$ and $B(s)$.

convergence is uniform in any compact interval of $S_{0}$. Notice $B(s)$ is increasing and differentiation of $A(s)$ is $(B(s)-A(s)) / s$. By $(\mathrm{J} 3)$, we have $A(s)>B(s)$ on $\left(0, s_{0}^{+}\right)$. Thus, $A(s)$ is decreasing for $0<s<s_{0}^{+}$. Also, $B(s)$ is increasing on $S_{0}$. Recall that we require that $B\left(s_{0}^{+}\right)=c_{0}^{+}$for some $s_{0}^{+}$and thus $B(0)<c_{0}^{+}$. Thus, for some $\delta>0$ and arbitrarily chosen $c \in\left(B(0), c_{0}^{+}\right)$we have

$$
B\left(s_{1}\right)+\delta<c<B\left(s_{2}\right)-\delta \text { and }|\mathbb{B}(s, \tau)-B(s)| \leq \delta
$$

for sufficiently small $\tau$ and appropriately chosen $s_{1}$ and $s_{2}$. Thus there exists an $s(\tau)$ such that $B(s(\tau), \tau)=c$. We can therefore choose $\tau$ small enough to get $c=\mathbb{B}(s(\tau), \tau)<\mathbb{A}(s(\tau), \tau)$ so that (A.9)-(A.11) are satisfied.

Define

$$
V(\xi)=V_{0}(\xi) I\{\xi \in(-\pi / \tau, 0)\} .
$$

By Lemma 2 in [2], it follows that $L_{c}[V]>0$ on $\xi \in(0, \pi / \tau)$. Now, we show that $Q_{c}\left[\epsilon V_{0}\right]$ is positive on $(-\pi / \tau, 0)$ for sufficiently small $\epsilon>0$. By the continuity of (A.8), we can choose $\varepsilon_{1}, \varepsilon_{2}>0$ such that for any $c \in\left(c_{0}^{-}, c_{0}^{+}\right), c$ is also in $\left(c_{0}^{-}\left(d_{1}^{*}, d_{2}^{*}\right), c_{0}^{+}\left(d_{1}^{*}, d_{2}^{*}\right)\right)$ where $\left(d_{1}^{*}, d_{2}^{*}\right):=\left(1+c w-\varepsilon_{1}, 1+\beta w+\varepsilon_{2}\right)$. Then we can choose $\epsilon$ small enough to get $Q_{c}\left[\epsilon V_{0}\right]-L_{c}\left[\epsilon V_{0}\right]=\varepsilon_{1} u+\varepsilon_{2}(J * u)+O\left(\epsilon^{2}\right)>0$. Thus, $Q_{c}[\epsilon V]>L_{c}[\epsilon V]>0$ on $(-\pi / \tau, 0)$. It follows from Theorem A.2 and above discussion that $Q_{c}(\epsilon V) \geq 0$.

Suppose $u$ is the solution to (3.7) and (3.13) with initial data $u_{0}$, then

$$
u(x, t)=u_{0}(x) e^{-(1+\beta w) t}+\int_{0}^{t} e^{-(1+\beta w)(t-s)} \mathcal{F}(u,(J * u))(x, s) d s
$$

and

$$
u(\xi, t)=u_{0}(\xi-c t) e^{-(1+\beta w) t}+\int_{0}^{t} e^{-(1+\beta w)(t-s)} \mathcal{F}(u,(J * u))(\xi-c(t-s), s) d s .
$$

Proposition A.2. Suppose Conditions (J1)-(J2) and (P1) hold. Then, the solution to

$$
\left\{\begin{array}{l}
\partial u / \partial t=Q_{c}[u], \\
u(\xi, 0)=\epsilon V
\end{array}\right.
$$

satisfies $\lim _{t \rightarrow \infty} u(\xi, t)=e$ or 1 for $c \in\left(c_{0}^{-}, c_{0}^{+}\right)$, where $V$ is the function defined in Lemma A.2. 
Proof. Let $X=u$ and $Y=\epsilon V(\xi)$ then $X(\xi, 0)=Y(\xi, 0)$ and

$$
\frac{\partial X}{\partial t}-Q_{c}[X]=0 \geq-Q_{c}[\epsilon V]=\frac{\partial Y}{\partial t}-Q_{c}[Y]
$$

By comparison principle, $X(\xi, t) \geq \epsilon V$. Applying comparison principle to $X$ and $Y$ with $X(\xi, 0)=u(\xi, 0)$ and $Y(\xi, 0)=u(\xi, h)$ for $h>0$, we can conclude that $u$ is non-decreasing function of $t$. By Theorem A.2, $u(\xi, t) \leq 1$. Therefore, $\lim _{t \rightarrow \infty} u(\xi, t)=q(\xi)$ exists by monotone convergence theorem. Define $\theta=\xi-c(t-s)$ so that

$$
u(\xi, t)=\epsilon V(\xi-c t) e^{-(1+\beta w) t}+\frac{1}{c} \int_{-\infty}^{\xi} e^{-\frac{1+\beta w}{c}(\xi-\theta)} I_{[\xi-c t, \xi]} \mathcal{F}(u,(J * u)) d \theta,
$$

where $\mathcal{F}(u,(J * u))$ has arguments $(\theta, t+(\theta-\xi) / c)$. Let $t \rightarrow \infty$, we have

$$
q(\xi)=\frac{1}{c} \int_{-\infty}^{\xi} e^{-\frac{1+\beta w}{c}(\xi-\theta)} \mathcal{F}(q,(J * q))(\theta) d \theta .
$$

Differentiating (A.12) gives

$$
q^{\prime}=-\frac{1+\beta w}{c} q+\frac{1}{c} \mathcal{F}(q,(J * q))
$$

which is the steady state equation of (3.1) (as well as (3.7)).

Equation (A.13) has three constant solutions, $0, e$ and 1. By Lemma A.2, we can conclude that $q(\xi)>\epsilon V$ on $[0, \pi / \tau]$. By the continuity of $q$, we conclude that $q(\xi+h)>\epsilon V$ in $[0, \pi / \tau]$ for sufficiently small $|h|$. We see that $q(\xi+h) \geq q(\xi)$ in $[0, \pi / \tau]$ for small enough $|h|$ by comparison principle. This implies $q^{\prime}=0$. Proposition 1 of [2] implies that $q>0$ on $(0, \pi / \tau)$. Hence $q(\xi)=e$ or 1 .

Let $W$ be the solution to the initial value problem specified by (ii) in Corollary 2. By Proposition 1 of [2], there exists a time $T>0$ such that

$$
\min _{0 \leq \xi<\pi / \tau} W(\xi, T)=m>0 .
$$

Choose $\epsilon$ small enough such that $\epsilon V \leq m$ in $[0, \pi / \tau]$. Suppose $u(\xi, t)$ is the solution to the initial value problem in Proposition A.2. Then $u(\xi, 0) \leq W(\xi, T)$. It follows from the comparison principle that $u(\xi, t-T) \leq$ $W(\xi, t)$ for all $t$. Hence, $\lim _{t \rightarrow \infty} W(\xi, t)=e$ or 1 as $\lim _{t \rightarrow \infty} u(\xi, t)=e$ or 1 by Proposition A.2.

\section{Appendix B Extra simulation studies}

In this section, we present an extra simulation study of the proposed model to computationally explore the long term behavior when the initial data $u_{0}$ is less than the equilibrium $e$ in some intervals and larger than $e$ in some other intervals. In particular, we consider compactly supported initial data using the same parameter settings in Section 4.2. Figure B.1 displays results for $c=-2$ while the initial data is larger than the stable equilibrium $e$ on a compactly supported set (the bold solid blue line). Compared to Figure 5 (c), which suggests that the defectors take over the population, Figure B.1 suggests that the cooperators still sustain in the population. It also implies that the limiting equilibrium might be the nontrivial co-existence solution $e$ in general. Motivated from this interesting simulation, further theoretical studies for this scenarios will be pursued in future.

\section{References}

[1] Andreu-Vaillo, F., Mazón, J. M., Rossi, J. D., Toledo-Melero, J. J., 2010. Nonlocal diffusion problems. Vol. 165. American Mathematical Society. 


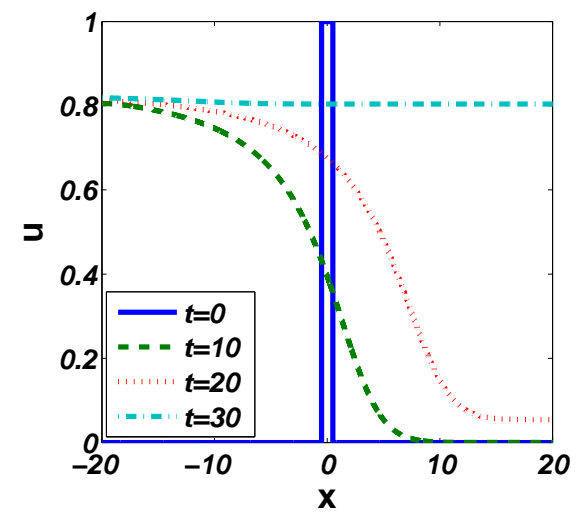

Figure B.1: Asymptotic behaviors of evolutionary spatial SD games with compactly supported initial data. Successive curves are separated by 10 time units, $\Delta x=0.002$ on $[-100,100]$ and $\Delta t=0.0005$.

[2] Aronson, D., 1977. The asymptotic speed of propagation of a simple epidemic. In: Nonlinear diffusion. Vol. 14. Pitman London, pp. 1-23.

[3] Aronson, D. G., Weinberger, H. F., 1975. Nonlinear diffusion in population genetics, combustion, and nerve pulse propagation. In: Partial differential equations and related topics. Springer, pp. 5-49.

[4] Axelrod, R., 2006. The evolution of cooperation: revised edition. Basic books.

[5] Benaïm, M., Weibull, J. W., 2003. Deterministic approximation of stochastic evolution in games. Econometrica $71(3), 873-903$.

[6] Brown, K., Carr, J., 1977. Deterministic epidemic waves of critical velocity. Math. Proc. Cambridge Philos. Soc 81 (3), 431-433.

[7] Celiker, H., Gore, J., 2013. Cellular cooperation: insights from microbes. Trends in cell biology 23 (1), 9-15.

[8] Chao, L., Levin, B. R., 1981. Structured habitats and the evolution of anticompetitor toxins in bacteria. Proc. Nat. Acad. Sci. 78 (10), 6324-6328.

[9] Chen, X., et al., 1997. Existence, uniqueness, and asymptotic stability of traveling waves in nonlocal evolution equations. Advances in Differential Equations 2 (1), 125-160.

[10] Colman, A. M., 2013. Game theory and its applications: in the social and biological sciences. Psychology Press.

[11] Cressman, R., 2003. Evolutionary dynamics and extensive form games. Vol. 5. MIT Press.

[12] Doebeli, M., Hauert, C., 2005. Models of cooperation based on the prisoner's dilemma and the snowdrift game. Ecology Letters 8 (7), 748-766.

[13] Durrett, R., Levin, S., 1997. Allelopathy in spatially distributed populations. Journal of theoretical biology 185 (2), 165-171.

[14] Falster, D. S., Westoby, M., 2003. Plant height and evolutionary games. Trends in Ecology \&amp; Evolution 18 (7), 337-343. 
[15] Fang, J., Wei, J., Zhao, X.-Q., 2008. Spatial dynamics of a nonlocal and time-delayed reaction-diffusion system. Journal of Differential Equations 245 (10), 2749-2770.

[16] Fang, J., Zhao, X.-Q., 2014. Traveling waves for monotone semiflows with weak compactness. SIAM Journal on Mathematical Analysis 46 (6), 3678-3704.

[17] Ferriere, R., Michod, R. E., et al., 2000. Wave patterns in spatial games and the evolution of cooperation. The Geometry of Ecological Interactions: Simplifying Spatial Complexity, 318-339.

[18] Fife, P. C., 2013. Mathematical aspects of reacting and diffusing systems. Vol. 28. Springer Science \&amp; Business Media.

[19] Fisher, R. A., 1930. The genetical theory of natural selection: a complete variorum edition. Oxford University Press.

[20] Helbing, D., Yu, W., 2009. The outbreak of cooperation among success-driven individuals under noisy conditions,. Proc. Nat. Acad. Sci. 106, 3680-3685.

[21] Hofbauer, J., 1998. Equilibrium selection via travelling waves. In: Game theory, experience, rationality. Springer, pp. 245-259.

[22] Hofbauer, J., Hutson, V., Vickers, G. T., 1997. Travelling waves for games in economics and biology. Nonlinear Analysis: Theory, Methods \& Applications 30 (2), 1235-1244.

[23] Hofbauer, J., Sigmund, K., 1998. Evolutionary games and population dynamics. Cambridge university press.

[24] Hsu, S.-B., Zhao, X.-Q., 2008. Spreading speeds and traveling waves for nonmonotone integrodifference equations. SIAM Journal on Mathematical Analysis 40 (2), 776-789.

[25] Huang, Y., Hao, Y., Wang, M., Zhou, W., Wu, Z., 2015. Optimality and stability of symmetric evolutionary games with applications in genetic selection. Mathematical Biosciences and Engineering 12 (3), $503-523$.

[26] Huang, Y., Wu, Z., 2012. Game dynamic model for yeast development. Bulletin of mathematical biology $74(7), 1469-1484$.

[27] Hutson, V., Vickers, G. T., 1992. Travelling waves and dominance of ess's. Journal of Mathematical Biology 30 (5), 457-471.

[28] Hwang, S., 2011. Spatial evolutionary game theory: Deterministic approximations, decompositions, and hierarchical multi-scale models. Ph.D. thesis, University of Massachusetts - Amherst.

[29] Hwang, S.-H., Katsoulakis, M., Rey-Bellet, L., 2013. Deterministic equations for stochastic spatial evolutionary games. Theoretical Economics 8 (3), 829-874.

[30] Kirkup, B. C., Riley, M. A., 2004. Antibiotic-mediated antagonism leads to a bacterial game of rockpaper-scissors in vivo. Nature 428 (6981), 412-414.

[31] Kot, M., Lewis, M. A., van den Driessche, P., 1996. Dispersal data and the spread of invading organisms. Ecology 77 (7), 2027-2042.

[32] Li, B., Weinberger, H. F., Lewis, M. A., 2005. Spreading speeds as slowest wave speeds for cooperative systems. Mathematical biosciences 196 (1), 82-98. 
[33] Liang, X., Zhao, X.-Q., 2007. Asymptotic speeds of spread and traveling waves for monotone semiflows with applications. Communications on pure and applied mathematics 60 (1), 1-40.

[34] Lui, R., 1989. Biological growth and spread modeled by systems of recursions. i. mathematical theory. Mathematical Biosciences 93 (2), 269-295.

[35] Lutscher, F., Pachepsky, E., Lewis, M. A., 2005. The effect of dispersal patterns on stream populations. Siam Review 47 (4), 749-772.

[36] Maynard Smith, J., 1974. The theory of games and the evolution of animal conflicts. Journal of theoretical biology 47 (1), 209-221.

[37] Maynard Smith, J., Price, G., 1973. The logic of animal conflict. Nature 246, 15.

[38] Maynard Smith, J., Szathmary, E., 1997. The major transitions in evolution. Oxford University Press.

[39] Medlock, J., Kot, M., 2003. Spreading disease: integro-differential equations old and new. Mathematical Biosciences 184 (2), 201-222.

[40] Mollison, D., 1991. Dependence of epidemic and population velocities on basic parameters. Mathematical biosciences 107 (2), 255-287.

[41] Neubert, M. G., Caswell, H., 2000. Demography and dispersal: calculation and sensitivity analysis of invasion speed for structured populations. Ecology 81 (6), 1613-1628.

[42] Nowak, M. A., 2006. Evolutionary dynamics. Harvard University Press.

[43] Nowak, M. A., Bonhoeffer, S., May, R. M., 1994. More spatial games. International Journal of Bifurcation and Chaos 4 (01), 33-56.

[44] Nowak, M. A., May, R. M., 1992. Evolutionary games and spatial chaos. Nature 359 (6398), 826-829.

[45] Nowak, M. A., Sigmund, K., et al., 2000. Games on grids. The Geometry of Ecological Interactions: Simplifying Spatial Complexity, 135-150.

[46] O. Aydogmus, W. Zhou, Y. K., Wu, Z., ???? Evolutionary spatial games with gaussian structures. Preprint.

[47] Ohtsuki, H., Hauert, C., Lieberman, E., Nowak, M. A., 2006. A simple rule for the evolution of cooperation on graphs and social networks. Nature 441 (7092), 502-505.

[48] Perthame, B., Souganidis, P. E., 2005. Front propagation for a jump process model arising in spacial ecology. Discrete and Continuous Dynamical Systems 13 (5), 1235-1246.

[49] Press, W. H., Dyson, F. J., 2012. Iterated prisoners dilemma contains strategies that dominate any evolutionary opponent. Proc. Nat. Acad. Sci. 109 (26), 10409-10413.

[50] Schumacher, K., 1980. Travelling-front solutions for integro-differential equations. i. J. Reine Angew. Math 316, 54-70.

[51] Taylor, C., Fudenberg, D., Sasaki, A., Nowak, M. A., 2004. Evolutionary game dynamics in finite populations. Bulletin of mathematical biology 66 (6), 1621-1644.

[52] Taylor, P. D., Jonker, L. B., 1978. Evolutionary stable strategies and game dynamics. Mathematical biosciences 40 (1), 145-156. 
[53] Traulsen, A., Claussen, J. C., Hauert, C., 2005. Coevolutionary dynamics: from finite to infinite populations. Physical review letters 95 (23), 238701.

[54] Turner, P. E., Chao, L., 1999. Prisoner's dilemma in an rna virus. Nature 398 (6726), 441-443.

[55] Weibull, J. W., 1997. Evolutionary game theory. MIT press.

[56] Weinberger, H. F., 1980. Some deterministic models for the spread of genetic and other alterations. In: Biological Growth and Spread. Springer, pp. 320-349.

[57] Weng, P., Zhao, X.-Q., 2006. Spreading speed and traveling waves for a multi-type sis epidemic model. Journal of Differential Equations 229 (1), 270-296.

[58] Young, H. P., 2001. Individual strategy and social structure: An evolutionary theory of institutions. Princeton University Press. 